\title{
Stability analysis of two-dimensional pool-boiling systems
}

\author{
M. Speetjens, A. Reusken† S. Maier-Paape $;$ W. Marquardt ${ }^{\S}$
}

February 28, 2007

RWTH Aachen, Templergraben 55, D-52056 Aachen, Germany.

\begin{abstract}
In this paper we consider a model for pool-boiling systems known from the literature. This model involves only the temperature distribution within the heater and models the heat exchange with the boiling medium via a nonlinear boundary condition imposed on the fluid-heater interface. The model allows multiple homogeneous (i.e. spatially constant) and multiple heterogeneous steady-state solutions. The structure of this family of steady-state solutions has been studied by means of a bifurcation analysis in two recent papers (Speetjens et al. (2006a), Speetjens et al. (2006b)). The present study concentrates on stability properties of these steady-state solutions. To this end, a generic linear and a case-specific nonlinear stability analysis are performed which show that only the homogeneous steady-states of complete nucleate or complete film boiling are linearly stable. All heterogeneous steady-state solutions appear linearly unstable. These stability results are consistent with laboratory observations.
\end{abstract}

PACS 44.35.+c, 44.05.+e, 02.60.Cb, 02.70.Jn

Keywords. pool boiling, stability, bifurcation analysis, numerical simulation

\section{Introduction}

Pool boiling refers to boiling processes that lean on natural convection as a means for heat transfer through the boiling medium and is the key mode of thermal transport in many practical applications. Local heat-transfer phenomena near heating walls in industrial boiling equipment (e.g. evaporators and kettle reboilers) for instance are essentially pool-boiling processes (Thome (2003)). Furthermore, pool boiling is emerging as novel cooling technique for electronics components (Mudawar (2001)). Despite its importance, many aspects of (pool)

\footnotetext{
* Laboratory for Energy Technology, Eindhoven University of Technology. This author was funded by the German Research Foundation through the Research Training Group "Hierarchy and Symmetry in Mathematical Models".

${ }^{\dagger}$ Corresponding author: Chair of Numerical Analysis, RWTH Aachen. Email: reusken@igpm.rwthaachen.de

${ }^{\ddagger}$ Chair of Mathematics, RWTH Aachen

${ }^{\S}$ Chair of Process Systems Engineering, RWTH Aachen
} 
boiling remain largely unexplored to date, mainly due to the immense complexity of the process induced by the intricate interplay between hydro- and thermodynamics. Studies on boiling known in the literature are mainly experimental and empirical. Theoretical investigations of fundamental phenomena in pool boiling, on the other hand, are scarce. This is the primary motivation for our recent studies, reported in Speetjens et al. (2006a) and Speetjens et al. (2006b), as well as for the follow-up study presented in this paper.

Central topic of the present study is the stability behaviour of pool-boiling systems. Laboratory experiments indicate that, without active control, pool-boiling systems allow only two stable steady-state solutions, namely nucleate boiling and film boiling (Adiutori (1964), Auracher \& Marquardt (2004), Kovalev (1968), Stephan (1965)). Other states belong to the transition-boiling regime and are inherently unstable. Nucleate boiling is, as opposed to film boiling, an efficient and safe mode of heat transfer and the sought-after boiling mode in most practical applications (Dhir (1998)). However, for typical operating conditions, the system admits both nucleate boiling and film boiling as steady states (Speetjens et al. (2006a)) and, consequently, the stable state eventually attained by the system is a priori unclear. Whether a given unstable state in the transition-boiling regime evolves towards either the nucleateboiling or the film-boiling state is of major practical importance, though. This is intimately related to the stability properties of boiling states. Stability analyses of pool-boiling systems are hitherto restricted to highly-idealised models as, for instance, heated wires (Gurevich \& Mints (1987), Kovalev \& Rybchinskaya (1978), Kovalev \& Usitakov (2003)), heated foils (Blum et al. (1999)), heated cylinders with homogeneous boiling conditions (Blum et al. (1996), Blum \& Marquardt (1998)) and rectangular 'thick' heaters with artificial heterogeneous boiling conditions (Blum et al. (1999)). Similar studies for more sophisticated models including both realistic heater geometries and realistic heterogeneous boiling conditions are not known in the literature. This is the impetus for the study presented in this paper.

The stability analysis in this paper concerns the stability properties of the multiple heterogeneous boiling states that have been found in Speetjens et al. (2006a) for a spatially two-dimensional (2D) heater. Key to the modelling approach is the phenomenological connection between the local state of aggregation of the boiling medium and the local temperature at the fluid-heater interface at mesoscopic length- and time scales ${ }^{1}$ : 'lower' and 'higher' temperatures correspond to the liquid and vapour phases, respectively. This allows a description of the (qualitative) behaviour of the pool-boiling problem entirely in terms of the temperature field within the heater. Thus the pool-boiling problem is reduced to a heat-transfer problem for the heater with a nonlinear heat-flux relation at the interface between the heater and the boiling medium. This heater-only model is based on the approach used in Blum et al. (1996), Blum et al. (1999). Section 2 provides a concise description. Further details can be found in Speetjens et al. (2006a).

The nonlinear heat-transfer model resembles nonlinear evolution equations of parabolic type (e.g. reaction-diffusion and pattern-formation equations) known from mathematical physics (Fife (1979), Temam (1997)). The dynamics of such systems are typically dominated by a global attractor, consisting of the steady-state solutions and their heteroclinic connections, to which initial conditions converge if time evolves (Temam (1997)). Said resemblance suggests that the pool-boiling model may exhibit similar dynamical behaviour. However, in the pool-boiling model the nonlinearity of the problem is due to the nonlinear heat-flux

\footnotetext{
${ }^{1}$ Here mesoscopic means locally averaged in space and time over intervals larger than bubble dimensions and bubble lifetimes in order to smooth out microscopic short-term fluctuations (Ouwekerk (1972)).
} 
condition at the heater-fluid interface and not due to a nonlinearity in the partial differential equation itself as in 'conventional' nonlinear parabolic evolution equations. Thus the concepts known for the latter problem class cannot be applied directly to the pool-boiling problem. We are unaware of rigorous mathematical studies on e.g. existence, smoothness and asymptotic stability of solutions of problems involving nonlinear boundary conditions such as our pool-boiling problem. A rigorous mathematical analysis is beyond the present scope, however. Instead, preliminary results are given that indicate the existence of a global attractor consisting of steady-state solutions and their heteroclinic connections (Section 3.1). This strongly suggests dynamical behaviour akin to that of 'conventional' nonlinear evolution equations and justifies a study on the existence of (multiple) steady-state solutions and their stability properties.

The set of steady-state solutions of the $2 \mathrm{D}$ boiling problem has been studied extensively in Speetjens et al. (2006a); an extension to the 3D case is given in Speetjens et al. (2006b). The present study investigates the stability properties of these steady states by a linear stability analysis. This analysis hinges on linearisation of the nonlinear problem at a given steady-state solution. Treatment of the resulting linearised model with a separation-of-variables technique results in a linear eigenvalue problem that governs the eigenmodes and corresponding eigenvalues (i.e. temporal growth rates) of steady-state solutions (Section 3.2.1). Analysis of this eigenvalue problem yields generic stability properties (Section 3.2.2). The eigenmodes and eigenvalues of a given steady state can be computed (approximately) using a Fouriercollocation discretisation method (Section 3.2.3). The generic linear-stability analysis and the eigenmode decomposition are demonstrated for a representative set of steady-state solutions (Section 4.1). A brief recapitulation of steady-state solutions determined in Speetjens et al. (2006a) is given in Section 3.3. Numerical simulation of the nonlinear evolution of linearly unstable steady states is performed by a spectral algorithm. These simulations validate the linear analysis and yield first insight into the nonlinear (in)stability behaviour (Section 4.2). Conclusions are drawn in Section 5.

\section{Model Problem}

The stability of pool-boiling systems is investigated in terms of a model problem considered in Speetjens et al. (2006a). An extensive discussion and motivation of this model is provided in the latter. Here we restrict ourselves to a concise description of the non-dimensional formulation of this model.

We consider the heat transfer within the two-dimensional rectangular heater $\mathcal{D}:=[0,1] \times$ $[0, D]$ (Figure 1a). Its boundary is given by $\Gamma=\partial \mathcal{D}=\Gamma_{H} \cup \Gamma_{A} \cup \Gamma_{F}$ and comprises the boundary segments $\Gamma_{H}: y=0$ (constant heat supply), $\Gamma_{A}: x=0,1$ (adiabatic sidewalls) and $\Gamma_{F}: y=D$ (non-constant heat extraction by boiling process). The heat transfer within $\mathcal{D}$ is modelled by

$$
\begin{aligned}
\frac{\partial T}{\partial t} & =\kappa \nabla^{2} T & & \text { in } \mathcal{D} \times\left[0, t_{\text {end }}\right], \\
T(\boldsymbol{x}, 0) & =T_{0}(\boldsymbol{x}) & & \text { for } \boldsymbol{x}:=(x, y) \in \mathcal{D}, \\
\frac{\partial T}{\partial \nu} & =g(\boldsymbol{x}, T) & & \text { on } \Gamma,
\end{aligned}
$$

with $\nu$ the outward normal of $\Gamma$. The boundary condition on $\Gamma$ introduces a nonlinearity due to the dependence of $g$ on $T(x, D, t)=: T_{F}(x, t)$. Note that $T_{F}$ denotes the value of $T$ at the 
fluid-heater interface $\Gamma_{F}$. The function $g$ is given by

$$
g(\boldsymbol{x}, Z)=\left\{\begin{aligned}
0 & \text { for } x=0 \text { or } x=1, \\
1 / \Lambda & \text { for } y=0, \\
-\Pi_{1} q_{F}\left(Z ; \Pi_{2}, \Pi_{3}\right) / \Lambda & \text { for } y=D .
\end{aligned}\right.
$$

The function $T=T(\boldsymbol{x}, t)$ is the non-dimensional temperature excess (i.e. the temperature relative to the boiling point of the medium). System parameters are $D$ (aspect ratio of the heater), $\Lambda$ (non-dimensional thermal conductivity), $\kappa$ (non-dimensional thermal diffusivity), and $\Pi_{1}$ (ratio typical boiling heat flux to constant heat supply), all of which are positive. The nonlinear heat-flux function $q_{F}\left(\cdot ; \Pi_{2}, \Pi_{3}\right)$ accounts for the heat exchange between the heater and the boiling medium. This function is specified in the Appendix and introduces two further control parameters, viz. $\Pi_{2}$ and $\Pi_{3}$, resulting in six parameters in total. However, physical considerations suggest $\Lambda D / \kappa=\left|1-\Pi_{1}\right|$ and thus the model contains five independent control parameters.

Physical considerations further suggest that the heat-flux function $q_{F}$, which describes the local heat exchange between fluid and heater, should be qualitatively similar to the socalled boiling curve. The latter describes the global heat exchange between fluid and heater obtained via averaging over the fluid-heater interface $\Gamma_{F}$. Therefore, as before (Speetjens et al. (2006a)) we use a heat-flux function schematically shown in Figure 1b, which has the typical shape of a boiling curve, cf. Appendix for an explicit expression. The heatflux function is parameterised by $\Pi_{2}$ (ratio of extremal heat fluxes) and $\Pi_{3}$ (ratio of extremal temperatures) and consists of three distinct regimes that correspond to one of the local boiling modes and associated mesoscopic states: nucleate boiling (left of local maximum; fluid-rich state); transition boiling (in between both extrema; transitional state); film boiling (right of local minimum; vapour-rich state). ${ }^{2}$
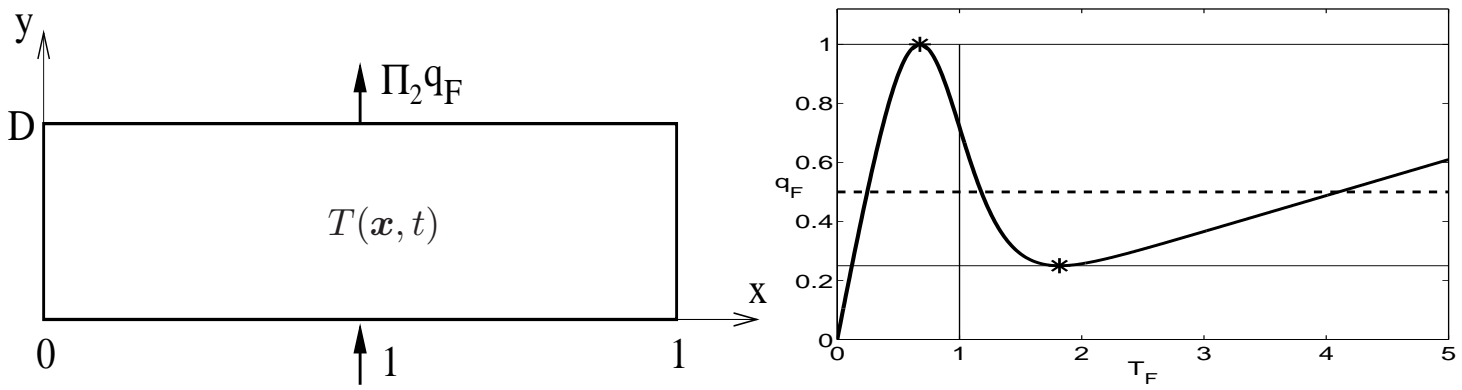

a) Heater configuration.

b) Heat-flux function.

Figure 1: Non-dimensional model problem: heater configuration (panel a) and heat-flux function $q_{F}$ (panel b). The dashed line represents the normalised heat supply $\Pi_{1}^{-1}$.

\section{Unsteady pool-boiling problem: generic analysis}

The nonlinear heat-transfer model (1) bears resemblance to nonlinear parabolic problems known from mathematical physics (Temam (1997)). However, a fundamental difference is that in the heat-transfer model (1) the nonlinearity resides in the boundary condition rather than

\footnotetext{
${ }^{2}$ Further physical background for this heater-only model is given by Speetjens et al. (2006a).
} 
in the PDE itself. This is a direct consequence of the finite thickness $(D>0)$ of the present heater configuration and distinguishes our problem from 'thin' $(D \rightarrow 0)$ configurations. For vanishing $D$ the model reduces to a PDE with a nonlinear source term (see e.g. Blum et al. (1999)) and generic concepts for the analysis of parabolic systems - in particular of the reaction-diffusion type (Fife (1979)) - can be applied. Examples of such approaches to poolboiling systems are in Gurevich \& Mints (1987), Kovalev \& Rybchinskaya (1978), Kovalev \& Usitakov (2003), Blum et al. (1999).

Rather than providing a rigorous mathematical analysis, which is beyond the present scope, in this section we indicate that concepts similar to those introduced for 'conventional' parabolic evolution equations (Temam (1997)) can be applied to the pool-boiling problem, too. We assume that solutions of the pool-boiling problem fulfill all the regularity conditions that admit application of such concepts. We note that the results of the generic analysis and those of the case study in Section 4 are in good agreement.

\subsection{Generic dynamical behaviour}

The dynamical behaviour of an evolutionary (parabolic) system is commonly investigated based on its corresponding weak formulation (Temam (1997)). We use the Sobolev space $H^{1}(\mathcal{D})$ and the notations

$$
(u, v)_{L^{2}(\mathcal{D})}:=\int_{\mathcal{D}} u v d x d y, \quad B(T, \varphi):=\int_{\mathcal{D}} \nabla T \cdot \nabla \varphi d x d y .
$$

For the heat-transfer problem (1) the weak formulation is as follows: find $u=u(t)=T(\cdot, t) \in$ $H^{1}(\mathcal{D})$ with $\frac{d u(t)}{d t} \in L^{2}(\mathcal{D})$ such that

$$
\left(\frac{d u(t)}{d t}, \varphi\right)_{L^{2}(\mathcal{D})}=\int_{\partial \mathcal{D}} g(\boldsymbol{x}, u) \varphi d \sigma-\kappa B(u, \varphi), \quad \text { for all } \varphi \in H^{1}(\mathcal{D}) .
$$

If $u$ is sufficiently smooth it follows (by taking test functions $\varphi \in C_{0}^{\infty}(\mathcal{D})$ ) that $d u / d t=\kappa \nabla^{2} u$ in $L^{2}(\mathcal{D})$. The boundary condition (in $L^{2}(\partial \mathcal{D})$ ) then follows from testing with $\left.\varphi \in C_{0}^{\infty}\left(\mathbb{R}^{2}\right)\right|_{\overline{\mathcal{D}}}$, and thus solutions of (3) provide $L_{2}$-solutions of (1). Conversely, regular solutions to (1) naturally are solutions to (3). The weak formulation describes the evolution of the system from its initial state $u(0)$ to its current state $u(t)$, i.e. $u(t)=\Phi_{t}(u(0))$ defines a flow. Thus the weak formulation (3) defines a dynamical system for the weak solution $u(t)$. Existence and uniqueness of a solution of (3) depends on smoothness and growth properties of $g$. This topic is not studied in the present paper. We assume that $g$ is such that the following property holds:

Property 1 System (3) results in a global semi-flow: $\Phi_{t}: H^{1}(\mathcal{D}) \rightarrow H^{1}(\mathcal{D}), \quad t \in[0, \infty)$.

The dynamical system (3) has a gradient structure, i.e. the functional $E: H^{1}(\mathcal{D}) \rightarrow \mathbb{R}$,

$$
E(u):=\frac{\kappa}{2} B(u, u)-\int_{\partial \mathcal{D}} G(\boldsymbol{x}, u) d \sigma,
$$

with $G(\boldsymbol{x}, u)$ such that $D_{u} G(\boldsymbol{x}, u)=g(\boldsymbol{x}, u)$, defines an energy or Lyapunov function for the solutions $u$ of $(3)$. Under certain smoothness and growth assumptions on $g$ we have $D E(u) \in \mathcal{L}\left(H^{1}(\mathcal{D}), \mathbb{R}\right)$ and

$$
D E(u)[\varphi]=\kappa B(u, \varphi)-\int_{\partial \mathcal{D}} g(\boldsymbol{x}, u) \varphi d \sigma, \text { for all } u, \varphi \in H^{1}(\mathcal{D}) .
$$


Along a solution curve $u(t)$ of (3) the energy $E$ decays monotonically in time:

$$
\frac{d}{d t}[E(u(t))]=D E(u)\left[\frac{d u}{d t}\right]=-\left(\frac{d u}{d t}, \frac{d u}{d t}\right)_{L^{2}(\mathcal{D})} \leq 0,
$$

with equality (for a range of $t$-values) only if $u(t)=T(\cdot, t)=T_{\infty}(\cdot)$ is a steady-state solution. This property implies a loss of energy of solutions $u(t)=T(\cdot, t)$ with progressing time. It is well known that in the presence of a Lyapunov function the omega limit set

$$
\omega\left(u_{0}\right)=\bigcap_{t>0} \overline{\bigcup_{r \geq t} \Phi_{r}\left(u_{0}\right)}
$$

of any point $u_{0} \in H^{1}(D)$ is a subset of the set of equilibria

$$
\mathcal{E}=\left\{u \in H^{1}(\mathcal{D}): \Phi_{t}(u)=u \text { for all } t \geq 0\right\} .
$$

The above already provides important information on the long-term dynamical behaviour by implying that any initial condition $u_{0}$ always converges on a steady-state solution.

Our case study for the pool-boiling problem further indicates the existence of a global attractor $\mathcal{A}$ for the semiflow $\Phi$., i.e. a strictly positive invariant subset of $H^{1}(\mathcal{D})$, which is compact and attracts all bounded subsets of $H^{1}(\mathcal{D})$ (cf. Temam (1997), Definition I. 1.3). We assume the following property to guarantee this existence:

Property 2 Assume the global semiflow $\Phi$. generated by (3) has some compactness property, e.g. in the sense of Temam (1997) (I. 1.12) or (I. 1.13), and further assume the existence of a bounded set $M \subset H^{1}(\mathcal{D})$, which attracts all bounded sets in $H^{1}(\mathcal{D})$.

In order to elucidate the consequences of the above properties we introduce the following notation: let the unstable set of a set $M \subset H^{1}(\mathcal{D})$ be defined as

$$
\begin{aligned}
\mathcal{M}_{u}(M):=\left\{w \in H^{1}(\mathcal{D}): \forall t \leq 0 \quad \exists u(t) \in\left(\Phi_{-t}\right)^{-1}(w)\right. & \text { and } \\
d(u(t), M) & \rightarrow 0 \text { for } t \rightarrow-\infty\}
\end{aligned}
$$

where $\left(\Phi_{s}\right)^{-1}(w):=\left\{v \in H^{1}(\mathcal{D}): \Phi_{s}(v)=w\right\}$ is the preimage of $w$ under $\Phi_{s}$ and $d(u, M)=\inf _{v \in M}\|u-v\|_{H^{1}(\mathcal{D})}$ is the distance between $u$ and $M$. Furthermore, we need the notion of a heteroclinic connection between two steady-state solutions $u_{0}$ and $v_{0}$. If $u_{0}, v_{0} \in \mathcal{E}$ are given, the heteroclinic connection between $u_{0}$ and $v_{0}$ is defined as

$$
\mathcal{C}\left(u_{0}, v_{0}\right):=\left\{w \in H^{1}(\mathcal{D}): w \in \mathcal{M}_{u}\left(u_{0}\right) \text { and } \Phi_{t}(w) \rightarrow v_{0} \text { as } t \rightarrow \infty\right\},
$$

i.e. all full orbits that approach $u_{0}$ as $t \rightarrow-\infty$ and $v_{0}$ as $t \rightarrow \infty$. Using these notions we can formulate the following result:

Theorem 1 We assume property 1 and 2. Then the semiflow $\Phi$. possesses a global compact attractor $\mathcal{A}$. This global attractor equals the unstable set of $\mathcal{E}$, i.e. $\mathcal{A}=\mathcal{M}_{u}(\mathcal{E})$, and consists of full orbits which approach the set of steady-state solutions for $t \rightarrow-\infty$. If furthermore $\mathcal{E}$ is discrete, then the global attractor consists of steady-state solutions $u(t)=T(\cdot, t)=T_{\infty}(\cdot) \in$ $H^{1}(\mathcal{D})$ of $(3)$, i.e.,

$$
\kappa B\left(T_{\infty}, \varphi\right)=\int_{\partial \mathcal{D}} g\left(\boldsymbol{x}, T_{\infty}\right) \varphi d \sigma \text { for all } \varphi \in H^{1}(\mathcal{D})
$$


and their heteroclinic connections:

$$
\mathcal{A}=\left(\bigcup_{u_{0} \in \mathcal{E}}\left\{u_{0}\right\}\right) \cup \bigcup_{u_{0}, v_{0} \in \mathcal{E}} \mathcal{C}\left(u_{0}, v_{0}\right),
$$

Proof. Temam (1997), Theorem I.1.1 and Theorem VII.4.1.

In general unstable steady-state solutions $u_{0}$ have a stable manifold

$$
\mathcal{M}_{s}\left(u_{0}\right)=\left\{w \in H^{1}(\mathcal{D}): \Phi_{t}(w) \rightarrow u_{0} \quad \text { for } \quad t \rightarrow \infty\right\}
$$

with a finite non-zero codimension. Therefore it is most likely for a generic initial condition $u_{0}$ to lie in the stable manifold of a stable equilibrium $g \in \mathcal{E}$, i.e. $\omega\left(u_{0}\right)=\{g\}$. Therefore in realistic systems due to physical imperfections as well as numerical simulations due to rounding errors the evolution process basically always converges towards a stable steady state.

Isolation of the global attractor of the boiling problem requires identification of its steadystate solutions and determination of the corresponding stability properties. The steady-state solutions have been studied extensively in Speetjens et al. (2006a); a brief recapitulation is given in Section 3.3. The present paper concerns the corresponding stability properties. Section 3.2 gives a generic linear stability analysis; Section 4 demonstrates and validates this linear analysis by way of numerical simulation of the nonlinear evolution of unstable steady-state solutions. This also offer first insight into the nonlinear stability behaviour of the system. Moreover, it may enable a more detailed investigation of the structure of the attractor by the approach proposed in Mischaikow (1995) along the lines of the analysis of the Cahn-Hilliard equation in Maier-Paape \& Miller (2002) and Maier-Paape et al. (2005).

\subsection{Linear stability analysis of steady-state solutions}

\subsubsection{Linearised heat-transfer model}

The stability analysis of steady-state solutions that we present is based on the linear theory of stability, cf. Iooss \& Joseph (1990) Section I.6. To determine stability of a steady-state solution $T_{\infty}(\boldsymbol{x})$ one subjects this steady-state solution to a small initial perturbation $v_{0}(\boldsymbol{x})=$ $v(\boldsymbol{x}, 0)$. We assume that for the nonlinear problem (1) the principle of linearized stability holds (cf. Kielhöfer (2004)), i.e., solutions of the nonlinear problem in a neighbourhood of a steady-state solution $T_{\infty}$ and of the linearised problem (linearisation at $T_{\infty}$ ) have the same qualitative behaviour. This assumption justifies the analysis of stability properties of the nonlinear problem by means of a stability analysis of the linearised problem. In this paper, unless stated otherwise explicitly, the notions stable and unstable are always meant in the sense of this linear theory of stability.

For the stability analysis we introduce the linearisation of (1). Let $T_{\infty}(\boldsymbol{x})$ be a regular steady-state solution of $(1)$, i.e. $T_{\infty}$ satisfies the following Laplace equation with a nonlinear Neumann boundary condition

$$
\nabla^{2} T_{\infty}=0 \quad \text { in } \mathcal{D}, \quad \frac{\partial T_{\infty}}{\partial \nu}=g\left(\boldsymbol{x}, T_{\infty}\right) \quad \text { on } \Gamma=\partial \mathcal{D} .
$$


( $g$ as in (2)). Properties of the nonlinear steady-state problem (8) are derived in Speetjens et al. (2006a) and summarised in Section 3.3. The corresponding linearised problem at $T_{\infty}$ for the perturbation $v(\boldsymbol{x}, t)$ induced by an initial perturbation $v_{0}(\boldsymbol{x})$ is given by

$$
\begin{aligned}
\frac{\partial v}{\partial t} & =\kappa \nabla^{2} v & & \text { in } \mathcal{D} \times\left[0, t_{\text {end }}\right], \\
v(\boldsymbol{x}, 0) & =v_{0}(\boldsymbol{x}) & & \text { for } \boldsymbol{x} \in \mathcal{D}, \\
\frac{\partial v}{\partial \nu} & =f(\boldsymbol{x}) v & & \text { on } \Gamma .
\end{aligned}
$$

The Neumann boundary condition is given by

$$
f(\boldsymbol{x})=\left\{\begin{aligned}
0 & \text { on } \Gamma \backslash \Gamma_{F} \\
-\gamma(x) & \text { on } \Gamma_{F}
\end{aligned}\right.
$$

with

$$
\gamma(x)=\frac{\Pi_{1}}{\Lambda} \frac{d q_{F}}{d Z}\left(T_{F, \infty}(x)\right), \quad x \in[0,1],
$$

where $T_{F, \infty}(x):=T_{\infty}(x, D)$ is the steady-state temperature profile at the fluid-heater interface $\Gamma_{F}$. Thus the original nonlinear condition on $\Gamma_{F}$ simplifies to a standard linear Neumann condition with an $x$-dependent coefficient determined by the interface temperature $T_{F, \infty}(x)$ of the steady-state solution.

As ansatz (based on separation of variables) we seek solutions of (9) of the form

$$
v(\boldsymbol{x}, t)=\mathrm{e}^{-\kappa \mu t} \psi(\boldsymbol{x}) \text {. }
$$

Substitution of (12) into (9) leads to the following linear elliptic eigenvalue problem for $\psi$ :

$$
\begin{aligned}
\nabla^{2} \psi+\mu \psi & =0 & & \text { in } \mathcal{D}, \\
\frac{\partial \psi}{\partial \nu} & =0 & & \text { on } \Gamma \backslash \Gamma_{F}, \\
\frac{\partial \psi}{\partial \nu}+\gamma(x) \psi & =0 & & \text { on } \Gamma_{F} .
\end{aligned}
$$

The weak formulation of this eigenvalue problem is as follows: determine $\mu_{n} \in \mathbb{R}, \psi_{n} \in H^{1}(\mathcal{D})$ such that

$$
-B\left(\psi_{n}, \varphi\right)-\int_{\Gamma_{F}} \gamma \psi_{n} \varphi d \sigma+\mu_{n} \int_{\mathcal{D}} \psi_{n} \varphi d x d y=0 \quad \text { for all } \varphi \in H^{1}(\mathcal{D}) .
$$

The eigenpairs that solve this problem are denoted by $\left(\mu_{n}, \psi_{n}\right), n=1,2, \ldots$. The eigenfunctions are scaled such that $\left\|\psi_{n}\right\|_{L^{2}(\mathcal{D})}=1$. Note that whether for generic $\gamma\left(\right.$ e.g. $\left.\gamma \in L^{\infty}\left(\Gamma_{F}\right)\right)$ the eigenfunctions $\left(\psi_{n}\right)_{n \geq 1}$ form a complete orthogonal basis of $L^{2}(\mathcal{D})$ is not a priori clear. We do not study this topic in the present paper. Instead, we assume that these eigenfunctions span a space that is sufficiently large such that it makes sense to restrict the choice of the initial perturbations $v_{0}(\boldsymbol{x})$ to this space. We obtain the following representation for the unique solution $v$ of (9) induced by an initial perturbation $v_{0} \in \operatorname{span}\left\{\psi_{n} \mid n \geq 1\right\}$ :

$$
\begin{aligned}
& \text { Let } v_{0}(\boldsymbol{x})=\sum_{k=0}^{\infty} \eta_{k} \psi_{k}, \quad \text { with } \eta_{k}=\left(v_{0}, \psi_{k}\right)_{L^{2}(\mathcal{D})}, \\
& \text { then } v(\boldsymbol{x}, t)=\sum_{k=1}^{\infty} \eta_{k} \mathrm{e}^{-\kappa \mu_{k} t} \psi_{k}(\boldsymbol{x}) .
\end{aligned}
$$


Note that the eigenvalues $\mu_{k}$ depend (via $\gamma$ ) on $T_{\infty}$ but do not depend on the perturbation $v_{0}$. As a consequence of the representation (18) one obtains:

Corollary 1 The steady-state solution $T_{\infty}$ of (1) is linearly stable w.r.t. all perturbations of the form (17) if

$$
\mu_{k} \geq 0 \quad \text { for all } k \text {. }
$$

Conversely, at least one $\mu_{k}<0$ implies that $T_{\infty}$ is an unstable steady-state solution.

Thus condition (19) characterises the linear stability of steady-state solutions.

Remark 1 From (16) it follows that

$$
\mu_{n} \int_{\mathcal{D}} \psi_{n}^{2} d x d y=B\left(\psi_{n}, \psi_{n}\right)+\int_{\Gamma_{F}} \gamma \psi_{n}^{2} d \sigma \geq \int_{\Gamma_{F}} \gamma \psi_{n}^{2} d \sigma
$$

Therefore we obtain $\mu_{n} \geq 0$ if $\gamma \geq 0$ holds. It follows that $\gamma \geq 0$ on the fluid-heater interface $\Gamma_{F}$ is a sufficient condition for stability of the steady-state solution.

\subsubsection{Analysis of the eigenvalue problem}

The stability properties of steady-state solutions $T_{\infty}$ of $(1)$ are directly related to the eigenvalues of problem (13)-(15), cf. Corollary 1 . In this section we study this eigenvalue problem.

We use an approach based on Fourier analysis. First, for a fixed $\mu \in \mathbb{R}$ we consider the problem (13)-(14) and apply separation of variables to construct bivariate Fourier-modes that satisfy (13)-(14). Let $\phi(\boldsymbol{x})=\alpha(x) \beta(y)$. An elementary computation shows that $c \alpha(x) \beta(y)$, $c \in \mathbb{R} \backslash\{0\}$ solves (13)-(14) iff

$$
\alpha(x)=\cos (n \pi x), \quad \beta(y)=\cosh \left(\sqrt{(n \pi)^{2}-\mu} y\right), \quad n=0,1,2, \ldots
$$

Note that for $z<0$ we have $\cosh (\sqrt{z})=\cosh (i \sqrt{|z|})=\cos (\sqrt{|z|})$. We now make the ansatz that the whole solution space of (13)-(14) is obtained by superposition of these Fourier-modes, i.e., all solutions of (13)-(14) lie in the space

$$
S_{\mu}:=\left\{\psi(\boldsymbol{x})=\sum_{n=0}^{\infty} A_{n} \cos (n \pi x) \cosh \left(\sqrt{\alpha_{n, \mu}} y\right), \quad A_{n} \in \mathbb{R}, \alpha_{n, \mu}:=(n \pi)^{2}-\mu\right\} .
$$

If we take the boundary condition (15) into account, then the solutions of (13)-(15) form a subspace of $S_{\mu}$. A function $\phi(\boldsymbol{x})=\sum_{n=0}^{\infty} A_{n} \cos (n \pi x) \cosh \left(\sqrt{\alpha_{n, \mu}} y\right) \in S_{\mu}$ solves (15) iff

$$
\sum_{n=0}^{\infty} A_{n} \sqrt{\alpha_{n, \mu}} \sinh \left(\sqrt{\alpha_{n, \mu}} D\right) \cos (n \pi x)+\gamma(x) \phi(x, D)=0 \text { for all } x \in[0,1] .
$$

Thus the problem of finding the eigenvalues of (13)-(15) is transformed to the problem of finding $\mu$ such that the equation (20) has a nontrivial solution $\phi_{F}(x):=\phi(x, D)$. Note that the latter problem is spatially one-dimensional. Furthermore, for $z<0$ we have $\sqrt{z} \sinh (\sqrt{z})=-\sqrt{|z|} \sin (\sqrt{|z|})$ and thus for all $\alpha_{n, \mu} \in \mathbb{R}$ equation (20) is real. We will 
solve this equation using a univariate Fourier analysis. To this end some notation is introduced. Let $\mathcal{F}: L^{2}([0,1]) \rightarrow \ell^{2}$ be the Fourier transform :

$$
\mathcal{F}\left(\sum_{n=0}^{\infty} c_{n} \cos (n \pi \cdot)\right)=\left(c_{n}\right)_{n \geq 0}
$$

For $\mathbf{c}, \mathbf{d} \in \ell^{2}$ the elementwise multiplication is denoted by $\mathbf{c} * \mathbf{d}=\left(c_{n} d_{n}\right)_{n \geq 0}$. Furthermore, we write for $\phi \in S_{\mu}$ restricted to fluid-heater interface $\Gamma_{F}$ :

$$
\begin{aligned}
\phi_{F}(x) & :=\phi(x, D)=\sum_{n=0}^{\infty} A_{n} \cosh \left(\sqrt{\alpha_{n, \mu}} D\right) \cos (n \pi x)=\sum_{n=0}^{\infty} \widetilde{\phi}_{n} \cos (n \pi x), \\
\widetilde{\phi}_{n} & :=A_{n} \cosh \left(\sqrt{\alpha_{n, \mu}} D\right) .
\end{aligned}
$$

Using this, (20) can be rewritten as follows: find $\phi_{F}(x)=\sum_{n=0}^{\infty} \widetilde{\phi}_{n} \cos (n \pi x)$ such that

$$
\sum_{n=0}^{\infty} \sqrt{\alpha_{n, \mu}} \tanh \left(\sqrt{\alpha_{n, \mu}} D\right) \widetilde{\phi}_{n} \cos (n \pi x)+\gamma(x) \phi_{F}(x)=0 \text { for all } x \in[0,1] .
$$

For $z<0$ we have $\sqrt{z} \tanh (\sqrt{z})=-\sqrt{|z|} \tan (\sqrt{|z|})$. Define

$$
\mathbf{d}_{\mu} \in \ell^{2}, \quad\left(d_{\mu}\right)_{n}:=\sqrt{\alpha_{n, \mu}} \tanh \left(\sqrt{\alpha_{n, \mu}} D\right), \quad n=0,1, \ldots
$$

Then the problem (22) has the following compact formulation: find $\phi_{F}$ such that

$$
J_{\mu} \phi_{F}:=\mathcal{F}^{-1}\left(\mathbf{d}_{\mu} * \mathcal{F} \phi_{F}\right)+\gamma \phi_{F}=0 .
$$

In this formulation it is implicitly assumed that a solution $\phi_{F}$ is sufficiently smooth such that $\mathbf{d}_{\mu} * \mathcal{F} \phi_{F} \in \operatorname{range}(\mathcal{F})$. The linear operator $J_{\mu}$ is well-defined on a dense subspace of $L^{2}([0,1])$. We are interested in values for $\mu$ for which the equation (23) has a nontrivial solution $\phi_{F}$. Furthermore, we are interested in the sign of these eigenvalues $\mu$, as these determine the stability of corresponding steady-state solutions. In the analysis below we distinguish two cases: constant and non-constant $\gamma$. For $\gamma(x)=\gamma=$ constant the relevant properties of $\mu$ can be determined analytically. For the general case of a smooth but not necessarily constant function $\gamma(x)$ certain properties can still be derived analytically. However, for full insight we must resort to discretisation of (23) and study its properties via numerical computations. Note that, for brevity, $\phi$ hereafter refers to both the full and the boundary solutions; its meaning readily follows from the context.

\section{Homogeneous temperature on fluid-heater interface: constant $\gamma$}

If $\gamma(x)=\gamma$ is constant the following holds:

Theorem 2 There exist sequences $\left(z_{k}^{+}\right)_{k \in \mathbb{N}}$ with $z_{k}^{+} \in\left[(k-1) \pi,\left(k-\frac{1}{2}\right) \pi\right), k \geq 1$, and $\left(z_{k}^{-}\right)_{k \in \mathbb{N}}$ with $z_{1}^{-}<0, z_{k+1}^{-} \in\left[\left(k-\frac{1}{2}\right) \pi, k \pi\right), k \geq 1$ such that the following holds. If $(\mu, \phi)$, with $\phi \neq 0$, solves (23) then $\mu \in\left(\mu_{k, n}\right)_{k, n \in \mathbb{N}}$ with $\mu_{k, n}$ defined by:

$$
\begin{aligned}
& \mu_{k, n}=z_{k}^{+}+(n \pi)^{2} \quad \text { if } \gamma \geq 0, \\
& \mu_{k, n}=z_{k}^{-}+(n \pi)^{2} \quad \text { if } \gamma<0 .
\end{aligned}
$$

For all $k, n$, the pair $\mu=\mu_{k, n}, \phi(x)=\cos (n \pi x)$ is a solution of (23). 
Proof. Note that

$$
\begin{aligned}
& \mathcal{F}^{-1}\left(\mathbf{d}_{\mu} * \mathcal{F} \phi\right)+\gamma \phi=0 \\
\Leftrightarrow & \mathbf{d}_{\mu} * \mathcal{F} \phi+\gamma \mathcal{F} \phi=0 \\
\Leftrightarrow & \text { for all } n:\left(d_{\mu}\right)_{n}+\gamma=0 \text { or } \widetilde{\phi}_{n}=(\mathcal{F} \phi)_{n}=0 .
\end{aligned}
$$

Take an $n \in \mathbb{N}$ such that $\widetilde{\phi}_{n} \neq 0$. Then $\left(d_{\mu}\right)_{n}+\gamma=0$ must hold. We consider the equation

$$
\mu \rightarrow\left(d_{\mu}\right)_{n}+\gamma=\sqrt{\alpha_{n, \mu}} \tanh \left(\sqrt{\alpha_{n, \mu}} D\right)+\gamma=0, \quad \alpha_{n, \mu}=(n \pi)^{2}-\mu .
$$

Thus we look for the roots of the function $g(z):=\sqrt{z} \tanh (\sqrt{z} D)+\gamma$. For $z>0$ and $\gamma \geq 0$ the equation $g(z)=0$ has no solution. For $z>0$ and $\gamma<0$ there is a unique root $z^{*}>0$. Define $z_{1}^{-}:=-z^{*}$. This induces a corresponding $\mu_{1}:=z_{1}^{-}+(n \pi)^{2}$ that solves (26). For $z \leq 0$ we have $g(z)=-\sqrt{-z} \tan (\sqrt{-z} D)+\gamma$. An elementary analysis shows that for $\gamma \geq 0$ the equation $g(z)=0$ has negative roots $z^{*}$ with $-z^{*}=: z_{k}^{+} \in\left[(k-1) \pi,\left(k-\frac{1}{2}\right) \pi\right)$ for $k=1,2, \ldots$. For $\gamma<0$ the equation $g(z)=0$ has negative roots $z^{*}$ with $-z^{*}=: z_{k+1}^{-} \in\left[\left(k-\frac{1}{2}\right) \pi, k \pi\right)$ for $k=1,2, \ldots$. Due to $-z=-\alpha_{n, \mu}=\mu-(n \pi)^{2}$ we obtain corresponding solutions of (26): $\mu_{k}=z_{k}^{+}+(n \pi)^{2}, k=1,2, \ldots$, for the case $\gamma \geq 0$ and $\mu_{k}=z_{k}^{-}+(n \pi)^{2}, k=2,3, \ldots$, for the case $\gamma<0$. Combination of the results for the case $z>0$ and $z \leq 0$ reveals that all possible roots of (26) are given by (24)- (25). If we take $\widetilde{\phi}_{n}=1, \widetilde{\phi}_{m}=0$ for all $m \neq n$ then $\left(\mu_{k, n}, \phi\right)$ with $\phi(x)=\cos (n \pi x)$ solves $(23)$.

Remark 2 Relation (24) implies that for the case $\gamma \geq 0$ all eigenvalues $\mu=\mu_{k, n}$ of (13)-(15) are positive. Relation (25) yields that for the case $\gamma<0$ there always exists some integer $n_{0}>0$ such that $\mu_{1, n}<0$ for all $0 \leq n \leq n_{0}$. Thus Theorem 2 describes the linear stability properties of the pool-boiling problem in case of constant $\gamma$. Steady-state solutions for which $\gamma \geq 0$ holds are linearly stable (consistent with Remark 1) whereas steady-state solutions for which $\gamma<0$ holds are unstable.

Remark 3 Using Theorem 2 and the representation (21) we obtain that the eigenfunction corresponding to $\mu=\mu_{k, n}$ is given by $\phi(x, y)=\cos (n \pi x) \cosh \left(\sqrt{(n \pi)^{2}-\mu_{k, n}} y\right)$. Note that $(n \pi)^{2}-\mu_{k, n}=-z_{k}^{+}$for $\gamma \geq 0$ and $(n \pi)^{2}-\mu_{k, n}=-z_{k}^{-}$for $\gamma<0$. Hence, for $\gamma \geq 0$ an eigenfunction corresponding to $\mu=\mu_{k, n}$ is given by $\phi(x, y)=\cos (n \pi x) \cos \left(\sqrt{z_{k}^{+}} y\right)$ and for $\gamma<0$ we obtain $\phi(x, y)=\cos (n \pi x) \cosh \left(\sqrt{-z_{1}^{-}} y\right)$ if $k=1$ and $\phi(x, y)=\cos (n \pi x) \cos \left(\sqrt{z_{k}^{-}} y\right)$ if $k \geq 2$.

\section{Heterogeneous temperature on fluid-heater interface: non-constant $\gamma$}

The linear operator $J_{\mu}$ in $(23)$ is symmetric on its domain in $L^{2}([0,1])$. This allows a general analysis using real eigenvalues and energy arguments. We introduce the notation $I:=[0,1]$ and $\gamma_{\min }:=\min _{x \in I} \gamma(x)$. We derive a similar stability condition as in Remark 1:

Theorem 3 Assume that $\gamma_{\text {min }} \geq 0$ holds. Then for all solutions $(\mu, \phi)$ of $(23)$, with $\phi \neq 0$, we have $\mu \geq 0$.

Proof. If $\mu, \phi \neq 0$ satisfies (23) we attain

$$
\left(\mathcal{F}^{-1}\left(\mathbf{d}_{\mu} * \mathcal{F} \phi\right), \phi\right)_{L^{2}(I)}+(\gamma \phi, \phi)_{L^{2}(I)}=0
$$


Suppose $\mu<0$. Then $\alpha_{n, \mu}=(n \pi)^{2}-\mu>0$ for all $n$ and thus $\left(d_{\mu}\right)_{n}>0$ for all $n$. This implies, with $c_{n}:=\|\cos (n \pi x)\|_{L^{2}(I)}$,

$$
\left(\mathcal{F}^{-1}\left(\mathbf{d}_{\mu} * \mathcal{F} \phi\right), \phi\right)_{L^{2}(I)}=\sum_{n=0}^{\infty}\left(d_{\mu}\right)_{n} c_{n}^{2} \widetilde{\phi}_{n}^{2}>0 .
$$

Combined with $(\gamma \phi, \phi)_{L^{2}(I)} \geq \gamma_{\min }(\phi, \phi)_{L^{2}(I)} \geq 0$ this results in contradiction with $(27)$.

Below we derive properties of eigenvalues $\mu<0$, i.e. eigenvalues for which corresponding unstable stationary solutions exist. Due to Theorem 3 these exist only if $\gamma_{\min }<0$ holds.

Lemma 1 Let $\mu<0$ be such that $J_{\mu} \phi=0$ for a $\phi \neq 0$, i.e. (23) holds. Then $\mu \in\left[\mu^{*}, 0\right)$ holds, where $\mu^{*}<0$ is the unique solution of

$$
\lambda^{*}\left(\mu^{*}\right)=0, \quad \lambda^{*}(\mu)=\sqrt{-\mu} \tanh (\sqrt{-\mu} D)+\gamma_{\min } .
$$

Proof. Due to $\gamma_{\min }<0$ relation (28) has a unique solution. Through $J_{\mu} \phi=0$ we arrive at

$$
\left(\mathcal{F}^{-1}\left(\mathbf{d}_{\mu} * \mathcal{F} \phi\right), \phi\right)_{L^{2}(I)}+(\gamma \phi, \phi)_{L^{2}(I)}=0 .
$$

For $\mu<\mu^{*}$ and arbitrary $\phi \neq 0$ we have

$$
\begin{aligned}
\left(\mathcal{F}^{-1}\left(\mathbf{d}_{\mu} * \mathcal{F} \phi\right), \phi\right)_{L^{2}(I)} & \geq\left(d_{\mu}\right)_{0}\|\phi\|_{L^{2}(I)}^{2}=\sqrt{-\mu} \tanh (\sqrt{-\mu} D)\|\phi\|_{L^{2}(I)}^{2} \\
& >\sqrt{-\mu^{*}} \tanh \left(\sqrt{-\mu^{*}} D\right)\|\phi\|_{L^{2}(I)}^{2} \\
& =\gamma_{\min }\|\phi\|_{L^{2}(I)}^{2} \geq-(\gamma \phi, \phi)_{L^{2}(I)} .
\end{aligned}
$$

Hence (29) can not hold for $\mu<\mu^{*}$.

Lemma 1 implies that negative eigenvalues must lie in the finite interval $\mu \in\left[\mu^{*}, 0\right)$. The result in the following theorem characterises the number of negative eigenvalues in this interval.

Theorem 4 Let $\mu^{*} \leq \mu_{1}<\mu_{2}<\ldots<\mu_{s}<0$ be all $\mu_{i}$ such that $J_{\mu_{i}} \phi_{i}=0$ for some $\phi_{i} \neq 0$. Assume that for all $i$ the null space of $J_{\mu_{i}}$ is one-dimensional. Let $\Sigma_{J}$ be the number of strictly negative eigenvalues (the "index") of $J_{0}=: J$ and assume that for each of these eigenvalues the corresponding eigenspace is one-dimensional. Then $s=\Sigma_{J}$ holds.

Proof. Let $\lambda(\mu), v(\mu)$ be an eigenpair of $J_{\mu}$, i.e., $J_{\mu} v(\mu)=\lambda(\mu) v(\mu)$, with $\|v(\mu)\|_{L^{2}(I)}=1$. This implies

$$
\lambda(\mu)=(\lambda(\mu) v(\mu), v(\mu))_{L^{2}(I)}=\left(J_{\mu} v(\mu), v(\mu)\right)_{L^{2}(I)} .
$$

Differentiation w.r.t. $\mu$ is denoted by a prime. Using the symmetry of $J_{\mu}$ we obtain

$$
\begin{aligned}
\lambda^{\prime}(\mu) & =\left(J_{\mu}^{\prime} v(\mu), v(\mu)\right)_{L^{2}(I)}+2\left(J_{\mu} v(\mu), v^{\prime}(\mu)\right)_{L^{2}(I)} \\
& =\left(J_{\mu}^{\prime} v(\mu), v(\mu)\right)_{L^{2}(I)}+2 \lambda(\mu)\left(v(\mu), v^{\prime}(\mu)\right)_{L^{2}(I)} \\
& =\left(J_{\mu}^{\prime} v(\mu), v(\mu)\right)_{L^{2}(I)} .
\end{aligned}
$$


The last equality follows from differentiation of $\|v(\mu)\|_{L^{2}(I)}^{2}=1$ w.r.t. $\mu$. The linear operator $J_{\mu}^{\prime}$ is given by $J_{\mu}^{\prime} \phi=\mathcal{F}^{-1}\left(\hat{\mathbf{d}}_{\mu} * \mathcal{F} \phi\right)$, with

$$
\left(\hat{d}_{\mu}\right)_{n}=\frac{d}{d \mu}\left(d_{\mu}\right)_{n}=\frac{d}{d \mu}\left[\sqrt{(n \pi)^{2}-\mu} \tanh \left(\sqrt{(n \pi)^{2}-\mu} D\right)\right] .
$$

An elementary computation yields that $-c_{0} \leq\left(\hat{d}_{\mu}\right)_{n}<0$ holds, where $c_{0}$ is a constant independent of $n$ and $\mu$. Thus $-c_{0} \leq\left(J_{\mu}^{\prime} v(\mu), v(\mu)\right)_{L^{2}(I)}=\lambda^{\prime}(\mu) \leq 0$ holds for all $\mu \leq 0$. This means that $\lambda(\mu)$ is a decreasing function with a bounded derivative. For $J_{\mu_{i}} \phi_{i}=0, \phi_{i} \neq 0$ (with a 1D null space) this implies a unique eigenvalue curve $\lambda(\mu)$ which passes through $\mu_{i}$, i.e. $\lambda\left(\mu_{i}\right)=0$. Due to the monotonicity of $\lambda(\mu)$ this curve must intersect the negative $y$-axis, resulting in a corresponding negative eigenvalue of $J_{0}$. This implies $s \leq \Sigma_{J}$. Conversely, let $\xi$ be a negative eigenvalue of $J_{0}$. Then there is a unique eigenvalue curve $\lambda(\mu)$ with $\lambda(0)=\xi$. Due to Lemma 1 this curve must intersect the $\mu$-axis in the interval $\left[\mu^{*}, 0\right)$. Hence, a unique corresponding $\mu_{i} \in\left[\mu^{*}, 0\right)$ exists such that $\lambda\left(\mu_{i}\right)=0$. This implies $\Sigma_{J} \leq s$.

¿¿From theorem 4 it follows that steady-state solutions with $\gamma_{\min }<0$ and $\Sigma_{J}>0$ are always unstable. The necessary condition for instability $\gamma_{\min }<0$ is satisfied only if the fluid-heater temperature falls (at least locally) within the transition regime; this implies a fundamental relation between transition boiling and instability.

The results of Theorem 4 induce a method for computing the number of negative eigenvalues $\mu \in\left[\mu^{*}, 0\right)$. This number equals the number of negative eigenvalues of the operator $J=J_{0}$. For the general case of a smooth but non-constant $\gamma$ the eigenvalues of $J_{\mu}$ cannot be determined analytically. Thus we apply a discretisation method (Section 3.2.3) for numerical approximation. Using a sufficiently high resolution this allows us to determine the correct number of negative eigenvalues (i.e. the same number as for the continuous problem). Moreover, an accurate approximation of the negative continuous eigenvalues can be computed. The resolution needed is not very high due to the fact that the eigenfunctions corresponding to the negative eigenvalues are dominated by low-frequency modes. This is explained in the following remark.

Remark 4 Consider $\phi$ with $\|\phi\|_{L^{2}(I)}=1$ and $\xi<0$ such that $J \phi=\xi \phi$. Represent $\phi$ in the cosine basis as $\phi(x)=c_{0}+\sum_{n=1}^{\infty} c_{n} \sqrt{2} \cos (n \pi x)$ (with $\left.\|\phi\|_{L^{2}(I)}^{2}=\sum_{n=0}^{\infty} c_{n}^{2}=1\right)$. Then

$$
\left(\mathcal{F}^{-1}\left(\mathbf{d}_{0} * \mathcal{F} \phi\right), \phi\right)_{L^{2}(I)}+(\gamma \phi, \phi)_{L^{2}(I)}=\xi(\phi, \phi)_{L^{2}(I)}<0
$$

holds, and

$$
\sum_{n=0}^{\infty} c_{n}^{2} n \pi \tanh (n \pi)=\left(\mathcal{F}^{-1}\left(\mathbf{d}_{0} * \mathcal{F} \phi\right), \phi\right)_{L^{2}(I)}=-(\gamma \phi, \phi)_{L^{2}(I)} \leq\|\gamma\|_{L^{\infty}(I)}\|\phi\|_{L^{2}(I)}^{2}=\|\gamma\|_{L^{\infty}(I)} .
$$

Thus $c_{n}$ must become "smaller" for "larger" $n$, meaning that, in this sense, the eigenfunction $\phi$ is dominated by low-frequency modes.

\subsubsection{Discretisation method}

As explained above, the problem of finding eigenvalues of (13)-(15) has been transformed to the problem of finding $\mu$ such that the equation (23) has a nontrivial solution. If for some $\mu$ 
an eigenvalue $\lambda(\mu)$ of $J_{\mu}$ equals zero, this $\mu$ is an eigenvalue of (13)-(15) For a general smooth function $\gamma$ the eigenvalue curves $\lambda(\mu)$ of the operator $J_{\mu}$ can not be determined analytically. We introduce a discretisation method that is used to discretise $J_{\mu}$ and thus determine the eigenvalue curves approximately. We use a Fourier-collocation method (Canuto et al. (1987)): determine $\phi_{F}(x):=\sum_{n=0}^{N} \widetilde{\phi}_{n} \cos (n \pi x)$ such that

$$
\sum_{n=0}^{N}\left\{\sqrt{\alpha_{n, \mu}} \tanh \left(\sqrt{\alpha_{n, \mu}} D\right)+\gamma\left(x_{k}\right)\right\} \widetilde{\phi}_{n} \cos \left(n \pi x_{k}\right)=0, \quad \text { for all } 0 \leq k \leq N,
$$

holds, with $x_{k}=k / N, k=0, \ldots, N$ the collocation points. Note that this is a discrete version of the continuous problem in (22). The $N+1$ equations (30) for the $N+1$ unknowns $\widetilde{\phi}_{n}$ can be represented in a compact matrix-vector formulation. To this end we introduce some notation. Let $\phi=\left(\phi_{0}, \ldots, \phi_{N}\right)^{T}$ be the vector of nodal values $\phi_{n}:=\phi_{F}\left(x_{n}\right)$. The latter relate to the truncated Fourier spectrum $\widetilde{\phi}=\left(\widetilde{\phi}_{0}, \ldots, \widetilde{\phi}_{N}\right)^{T}$ via

$$
\phi=\boldsymbol{V} \widetilde{\boldsymbol{\phi}} \quad \widetilde{\boldsymbol{\phi}}=\boldsymbol{V}^{-1} \boldsymbol{\phi} \text { with } \boldsymbol{V}:=\left[\begin{array}{cccc}
1 & \cos \left(\pi x_{0}\right) & \ldots & \cos \left(N \pi x_{0}\right) \\
\vdots & \vdots & & \vdots \\
1 & \cos \left(\pi x_{N}\right) & \ldots & \cos \left(N \pi x_{N}\right)
\end{array}\right]=\boldsymbol{V}^{T} \text {. }
$$

The relation

$$
\boldsymbol{V}^{-1}=\frac{2}{N} \boldsymbol{D} \boldsymbol{V} \boldsymbol{D}, \quad \boldsymbol{D}=\operatorname{diag}\left(\frac{1}{2}, 1, \ldots, 1, \frac{1}{2}\right)
$$

holds, i.e. the matrix $\sqrt{\frac{2}{N}} \boldsymbol{V} \boldsymbol{D}$ is orthogonal. Define

$$
\begin{aligned}
\boldsymbol{K}_{\mu} & =\boldsymbol{V} \boldsymbol{K}_{S, \mu} \boldsymbol{V}^{-1}, \quad \boldsymbol{K}_{S, \mu}=\operatorname{diag}\left(\sqrt{\alpha_{n, \mu}} D \tanh \left(\sqrt{\alpha_{n, \mu}} D\right)\right)_{0 \leq n \leq N}, \\
\boldsymbol{Q} & =\operatorname{diag}\left(D \gamma\left(x_{n}\right)\right)_{0 \leq n \leq N} .
\end{aligned}
$$

Note that $\boldsymbol{K}_{S, \mu}$ and $\boldsymbol{Q}$ are both diagonal matrices. The discrete problem (30) can be formulated in matrix-vector form as

$$
\boldsymbol{J}_{\mu} \boldsymbol{\phi}=\mathbf{0}, \quad \boldsymbol{J}_{\mu}:=\boldsymbol{K}_{\mu}+\boldsymbol{Q} .
$$

In spectral form this becomes

$$
\boldsymbol{J}_{S, \mu} \widetilde{\boldsymbol{\phi}}=\mathbf{0}, \quad \boldsymbol{J}_{S, \mu}:=\boldsymbol{V}^{-1} \boldsymbol{J}_{\mu} \boldsymbol{V}=\boldsymbol{K}_{S, \mu}+\boldsymbol{Q}_{S}, \quad \boldsymbol{Q}_{S}:=\boldsymbol{V}^{-1} \boldsymbol{Q} \boldsymbol{V} .
$$

The eigenvalues $\mu$ and eigenfunctions $\phi_{F}$ are approximated by those $\mu \in \mathbb{R}$ and $\phi \in \mathbb{R}^{N+1}$ for which $\phi$ is a non-trivial null-vector of $\boldsymbol{J}_{\mu}$.

Numerical tests for the case study in Section 4 revealed that in these (approximate) eigenfunctions, for sufficiently high $N$, the Fourier coefficients decay exponentially with increasing wave number $n$. This is consistent with Remark 4 in that indeed the low-frequency modes are dominant. In all our experiments we use a resolution with $N=128$. This resolution allows a correct determination of the number of negative eigenvalues $\mu$ as well as an accurate approximation of their numerical value. 


\subsection{Steady-state solutions}

A detailed analysis of the steady-state behaviour of the pool-boiling problem is given in Speetjens et al. (2006a). The approach is in essence similar to that adopted above. The nonlinear $2 \mathrm{D}$ steady-state problem (8) is reduced to a $1 \mathrm{D}$ boundary model via the method of separation of variables. This 1D model is solved (approximately) through numerical approximation with a Fourier-collocation discretisation method. Below the issues relevant in the present context are summarised.

Application of separation of variables to (8) yields a (formal) representation of the solution of the Laplace equation and the linear Neumann boundary conditions on $\Gamma \backslash \Gamma_{F}$. This results in

$$
T_{\infty}(x, y)=\sum_{n=0}^{\infty} \widetilde{T}_{n} \frac{\cosh (n \pi y)}{\cosh (n \pi D)} \cos (n \pi x)+\frac{D-y}{\Lambda}
$$

which can easily be checked by substitution. The coefficients $\widetilde{T}_{n}$ form the spectrum of the Fourier cosine expansion

$$
T_{F, \infty}(x):=T_{\infty}(x, D)=\sum_{n=0}^{\infty} \widetilde{T}_{n} \cos (n \pi x)
$$

of the temperature profile at the fluid-heater interface $\Gamma_{F}$. These coefficients are determined by the nonlinear Neumann boundary condition on $\Gamma_{F}$. Substitution of (37) into the nonlinear boundary condition on $\Gamma_{F}$ leads to

$$
\sum_{n=0}^{\infty} n \pi \tanh (n \pi D) \widetilde{T}_{n} \cos (n \pi x)+\eta\left(T_{F, \infty}(x)\right) T_{F, \infty}(x)-\frac{1}{\Lambda}=0 \quad \text { for all } x \in[0,1],
$$

where $\eta\left(T_{F}\right)=\frac{\Pi_{1}}{\Lambda} \frac{q_{F}\left(T_{F}\right)}{T_{F}}$ is the scaled heat-transfer coefficient. The nonlinear equation (39) is the characteristic equation that determines the particular properties of the steady-state solutions of (8). Note the resemblance with relation (22).

The reduced steady-state problem (39) admits trivial and non-trivial solutions. Trivial solutions are homogeneous interface temperatures, for which $T_{F, \infty}(x)=\widetilde{T}_{0}$ and $\widetilde{T}_{n}=0$ for $n \geq 1$ holds. Then the nonlinear condition (39) simplifies to

$$
q_{F}\left(\widetilde{T}_{0}\right)=\Pi_{1}^{-1} .
$$

Thus homogeneous solutions coincide with intersection(s) between the heat-flux function $q_{F}$ and the normalised heat-supply given by $\Pi_{1}^{-1}$ (Figure $1 b$ ). Non-trivial, i.e., heterogeneous, solutions $T_{F, \infty}(x)$ that satisfy (39) cannot be determined analytically. However, certain properties of such solutions (if they exist) can be derived. One important property, proved in Speetjens et al. (2006a), is that such solutions always occur as conjugate pairs

$$
T_{F, \infty}(x) \text { and } T_{F, \infty}^{*}(x):=T_{F, \infty}(x+1 / k) \text { for a } k \in \mathbb{N} \text {. }
$$

This means that if $T_{F, \infty}(x)$ is a solution then (for a certain $k \in \mathbb{N}$ ) the shifted function $T_{F, \infty}(x+1 / k)$ is a solution, too. This implies non-uniqueness of heterogeneous solutions. Homogeneous steady-state solutions can easily be determined by a standard root-finding method applied to (40). Heterogeneous steady-state solutions are computed (approximately) 
by using a Fourier-collocation discretisation method, as described in section 3.2.3, applied to (39). This results in a nonlinear system of equations of the form

$$
\begin{aligned}
& \mathcal{G}(\boldsymbol{T}):=(\boldsymbol{K}+\boldsymbol{M}(\boldsymbol{T})) \boldsymbol{T}-\boldsymbol{G}=\mathbf{0}, \\
& \boldsymbol{T}=\left(T_{0}, \ldots, T_{N}\right)^{T}, T_{n}:=T_{F, \infty}\left(x_{n}\right), \boldsymbol{K}=\boldsymbol{K}_{0} \text { as in }(33) \text { and } \\
& \quad \boldsymbol{M}=\operatorname{diag}\left(\eta_{n}\right)_{0 \leq n \leq N}, \quad \eta_{n}:=\eta\left(T_{F, \infty}\left(x_{n}\right)\right), \quad \boldsymbol{G}=(1 / \Lambda, \ldots, 1 / \Lambda)^{T} .
\end{aligned}
$$

The discrete system (42) defines a nonlinear set of equations that is solved by a continuation procedure. To this end we introduce a parameterised heat-flux function

$$
q_{F}\left(T_{F} ; P\right):=C_{D}\left(F_{1}-P F_{2} H\left(C_{D} T_{F}-1\right)\right) T_{F}, \quad 0 \leq P \leq 1 .
$$

(cf. (48) in the Appendix). In this modified heat-flux function the degree of nonlinearity is controlled through the nonlinearity parameter $P$. For $P=0$ function (44) reduces to a linear form; for $P=1$ the physical heat-flux $q_{F}\left(T_{F}\right)$ is recovered. This $P$-dependence of $q_{F}$ induces a $P$-dependence of the matrix $M$ via the function $\eta$. This is expressed by the notation $M_{P}$. The continuation procedure is applied to the function

$$
P \rightarrow \mathcal{G}(\boldsymbol{T}, P):=\left(\boldsymbol{K}+\boldsymbol{M}_{P}(\boldsymbol{T})\right) \boldsymbol{T}-\boldsymbol{G}=\mathbf{0} .
$$

For each $P \in[0,1]$ the set of homogeneous solutions of this system can be easily computed. Starting on a branch of homogeneous solutions the continuation algorithm determines (pitchfork) bifurcations at which the conjugate solution pairs (41) branch off from the homogeneous branch. An extensive treatment of these bifurcation results is given in Speetjens et al. (2006a). In section 4.1 a bifurcation diagram for a representative case study is given.

\section{Unsteady boiling problem: an illustrative case study}

In this section the concepts introduced above are demonstrated by means of a representative case study. Unless indicated otherwise, the fixed parameter set $\Lambda=0.2, D=0.2, \Pi_{2}=4$, $\Pi_{1}=2$ and $\Pi_{3}=0.37$ (corresponding with $W=1$; see Appendix) is used. The steadystate solutions and corresponding linear stability properties are treated in Section 4.1. The nonlinear (long-term) evolution of perturbed unstable steady-state solutions is investigated in Section 4.2 via numerical simulation of (1). These results give a numerical validation of the linear stability analysis (Section 4.1) and provide insight into the nonlinear stability behaviour (Section 4.2).

\subsection{Steady-state solutions and their linear stability properties}

\subsubsection{Homogeneous steady-state solutions}

Homogeneous solutions are determined through relation (40) and coincide with the intersections between the heat-flux function $q_{F}$ (solid) and the normalised heat supply given by $\Pi_{1}^{-1}$ (dashed) in Figure 1b. Three non-degenerate situations can occur:

Regime $\boldsymbol{\Pi}_{\mathbf{1}}>\boldsymbol{\Pi}_{\mathbf{2}}$ : Relation (40) admits one steady-state solution $T_{F, \infty}$ in the nucleateboiling regime (Figure $2 a)$. The local positive slope of the boiling curve $\left(\dot{q}_{F}:=d q_{F} / d T>\right.$ $0)$ implies $\gamma>0$ and thus by Theorem 2 we have stability of $T_{F, \infty}$. 
Regime $1<\Pi_{1}<\Pi_{2}$ : Relation (40) yields three steady-state solutions (Figure $2 b$ ): $T_{F, \infty}^{(1)}$ (nucleate boiling); $T_{F, \infty}^{(2)}$ (transition boiling); $T_{F, \infty}^{(3)}$ (film boiling). For $T_{F, \infty}^{(1,3)}$ we have $\gamma>0$ (due to $\dot{q}_{F}>0$ ) and thus by Theorem 2 stability of these steady-state solutions. For $T_{F, \infty}^{(2)}$ we have $\dot{q}_{F}<0$ and thus $\gamma<0$. From Theorem 2 we conclude that $T_{F, \infty}^{(2)}$ is unstable.

Regime $\boldsymbol{\Pi}_{\mathbf{1}}<\mathbf{1}$ : Relation (40) has one steady-state solution $T_{F, \infty}$ in the film-boiling regime (Figure $2 c$ ). From $\gamma>0$ and Theorem 2 we conclude that this solution is stable.

Cases $\Pi_{1}=\Pi_{2}$ and $\Pi_{1}=1$ are the degenerate cases through which the system switches between one and three homogeneous solutions.

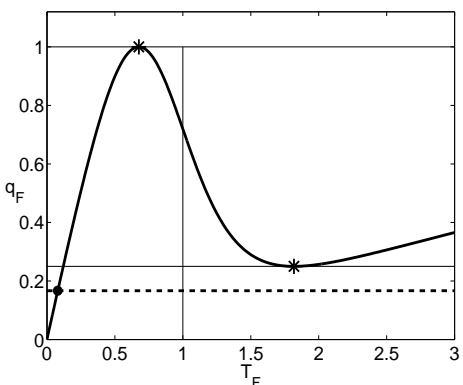

a) $\Pi_{1}>\Pi_{2}$

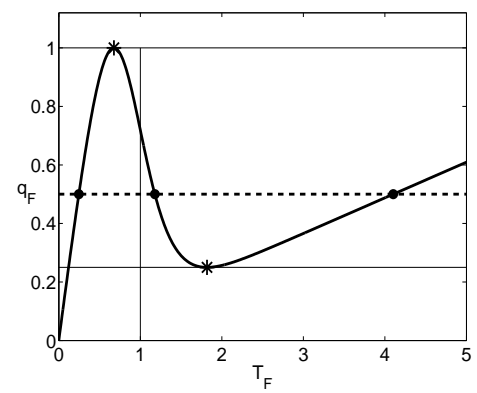

b) $1<\Pi_{1}<\Pi_{2}$

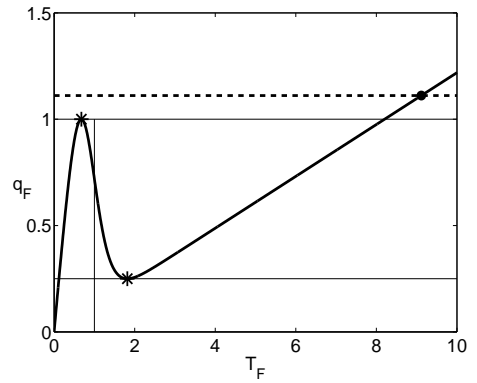

c) $\Pi_{1}<1$

Figure 2: Homogeneous steady-state solution(s) as a function of the system parameters $\Pi_{1}$ and $\Pi_{2}$. The solutions (dots) coincide with the intersections between the heat-flux function $q_{F}$ (solid) and normalised heat supply $\Pi_{1}^{-1}$ (dashed).

\subsubsection{Heterogeneous steady-state solutions}

Steady-state behaviour The study of Speetjens et al. (2006a) strongly suggests that heterogeneous solution pairs (41) emerge only from pitchfork bifurcations that occur on branches of homogeneous solutions. For homogeneous solutions, $\boldsymbol{T}=T_{F}=$ constant, the Jacobian w.r.t $T$ of $\mathcal{G}(\boldsymbol{T} ; P)$ is given by

$$
\frac{\partial \mathcal{G}(\boldsymbol{T} ; P)}{\partial \boldsymbol{T}}=: \hat{\boldsymbol{J}}_{P}(\boldsymbol{T})=\boldsymbol{V} \boldsymbol{\Lambda}_{P} \boldsymbol{V}^{-1}, \quad \boldsymbol{\Lambda}_{P}=\operatorname{diag}\left(n \pi \tanh (n \pi D)+\gamma_{P}\left(T_{F}\right)\right)_{0 \leq n \leq N}
$$

with $\gamma_{P}(Z)=\frac{\Pi_{1}}{\Lambda} \frac{\partial q_{F}(Z ; P)}{\partial Z}$. Note that for $P=1$ (i.e., $q_{F}(Z ; 1)=q_{F}(Z)$ ) this Jacobian is equal to the matrix $\boldsymbol{J}_{0}$ in (35): $\hat{\boldsymbol{J}}_{1}=\boldsymbol{J}_{0}$. The origin of this identity lies in the fact that for $\mu=0$ the linear eigenvalue problem (13)-(15) (which has a corresponding discrete boundary operator $\boldsymbol{J}_{0}$ as in (35)) is the same as the linearisation of the stationary problem in (8) (which has a corresponding discrete boundary operator $\hat{\boldsymbol{J}}_{1}$ as in (46)).

The eigenvalues and corresponding eigenvectors of $\hat{\boldsymbol{J}}_{P}$ are given by

$$
\lambda_{n}=n \pi \tanh (n \pi D)+\gamma_{P}\left(T_{F}\right), \quad \boldsymbol{v}_{n}=\left(\cos \left(n \pi x_{0}\right), \ldots, \cos \left(n \pi x_{N}\right)\right)^{T}, \quad 0 \leq n \leq N .
$$

The eigenvector $\boldsymbol{v}_{n}$ coincides with the $n$-th Fourier mode. The Jacobian is singular if one or more of its eigenvalues $\lambda_{n}$ vanish. Because $n \pi \tanh (n \pi D) \geq 0$ for all $n \geq 0$, this can only happen if $\gamma\left(T_{F}\right) \leq 0$. Thus a bifurcation on a homogeneous solution branch can only 
occur for those $T_{F}$ for which the boiling curve has a negative slope. Only the intersection $T_{F}^{(2)}$ satisfies this criterion. This explains why bifurcations are restricted to the $T_{F}^{(2)}$-branch in the bifurcation diagram (Figure 3). This implies that bifurcations - and thus multiple (heterogeneous) solutions - can only occur for surface temperature values in the transition range of the boiling curve. Figure 3 shows the bifurcation diagram as a function of the nonlinearity parameter $P$ (Speetjens et al. (2006a)). The heavy curves are the solution branches corresponding to the homogeneous solutions $T_{F, \infty}^{(1,2,3)}$. The lower (nearly-horizontal) branch coincides with the intersection $T_{F, \infty}^{(1)}$ that exists for all $0 \leq P \leq 1$; the upper branch, with turning point at $P_{B}$ (here $P_{B} \approx 0.926$ ), coincides with the two intersections $T_{F, \infty}^{(2,3)}$ that exist only in the interval $P_{B} \leq P \leq 1$. The lower and upper legs of this upper branch (connected at the turning point) correspond to $T_{F, \infty}^{(2)}$ and $T_{F, \infty}^{(3)}$, respectively. The solid curves are the heterogeneous solution branches that originate from pitchfork bifurcations (dots) on the $T_{F, \infty}^{(2)}$-branch and from left to right correspond with the single vanishing eigenvalue $\lambda_{n}=0$ for $n=1,2,3$. The corresponding eigenvector $\boldsymbol{v}_{n}$ determines the form of the bifurcating heterogeneous solution and equals the $n$-th Fourier mode. These heterogeneous solutions that originate from the bifurcation point corresponding to $\lambda_{n}$ are called 'mode- $n$ ' solutions. The lower and upper legs in a pitchfork bifurcation correspond to $T_{F, \infty}$ and its conjugate $T_{F, \infty}^{*}$, respectively, cf. (41).

The final states $(P=1)$ in the bifurcation diagram (Figure 3$)$ correspond with the physically meaningful steady-state solutions to the boiling problem (1). Figure 4 (top row) shows the boundary profiles $T_{F, \infty}$ associated with the pairs of steady-state mode- $n$ solutions, where solid and dashed lines indicate $T_{F, \infty}$ and its conjugate $T_{F, \infty}^{*}$, respectively. Figure 4 (bottom row) gives the profiles of the function $\gamma(x)$ from (11) corresponding to $T_{F, \infty}$.

\subsubsection{Qualitative linear stability properties}

Theorem 3 and Theorem 4 state that the qualitative stability (i.e. stable or unstable) of the heterogeneous mode- $n$ solutions depends on $\gamma_{\min }$ and the index $\Sigma_{J}$. The boundary profiles (Figure 4) show that for all cases we have $\gamma<0$ - and hence transition boiling - on one or more sections of the boundary $\Gamma_{F}$ (Figures $4 d-f$ ). This implies $\gamma_{\min }<0$ and thus rules out unconditional stability (Theorem 3); stability properties depend on the quantity $\Sigma_{J}$ as defined in Theorem 4 . We generalise the definition of $\Sigma_{J}$ by defining $\Sigma_{J}$ as the $P$-dependent number of negative eigenvalues of the Jacobian $\hat{\boldsymbol{J}}_{P}$ from (46). The identity $\hat{\boldsymbol{J}}_{1}=\boldsymbol{J}_{0}$ means that for $P=1$ this generalised $\Sigma_{J}$ coincides with the $\Sigma_{J}$ as defined Theorem 4 (provided that the resolution is high enough to determine the correct number of negative eigenvalues, cf. Remark 4). Thus the linear stability properties of the steady-state solutions follow directly from the bifurcation analysis visualised in Figure 3. On the homogeneous branches $\Sigma_{J}$ can be easily computed using (47). Computational analysis reveals that on the heterogeneous solution branches no further bifurcations, apart from the pitchfork bifurcation points at the intersection with the homogeneous branch, occur. Thus $\Sigma_{J}$ remains constant along a heterogeneous branch, meaning that $\Sigma_{J}$ for $P=1$ (the relevant quantity for Theorem 4) equals $\Sigma_{J}$ at the underlying bifurcation. The value for $\Sigma_{J}$ at $P=1$ is computed numerically. The values for $\Sigma_{J}$ corresponding with each solution branch are indicated in Figure 3. For all heterogeneous solutions we have $\Sigma_{J}>0$ at $P=1$ and thus, due to Theorem 4, all heterogeneous solutions are unstable. Moreover, $\Sigma_{J}$ - and thereby the number of unstable modes (Theorem 4) - increases with each bifurcation. In this sense mode- $n$ solutions become more unstable for higher $n$. The change of the value of $\Sigma_{J}$ at the bifurcation points and its effect 


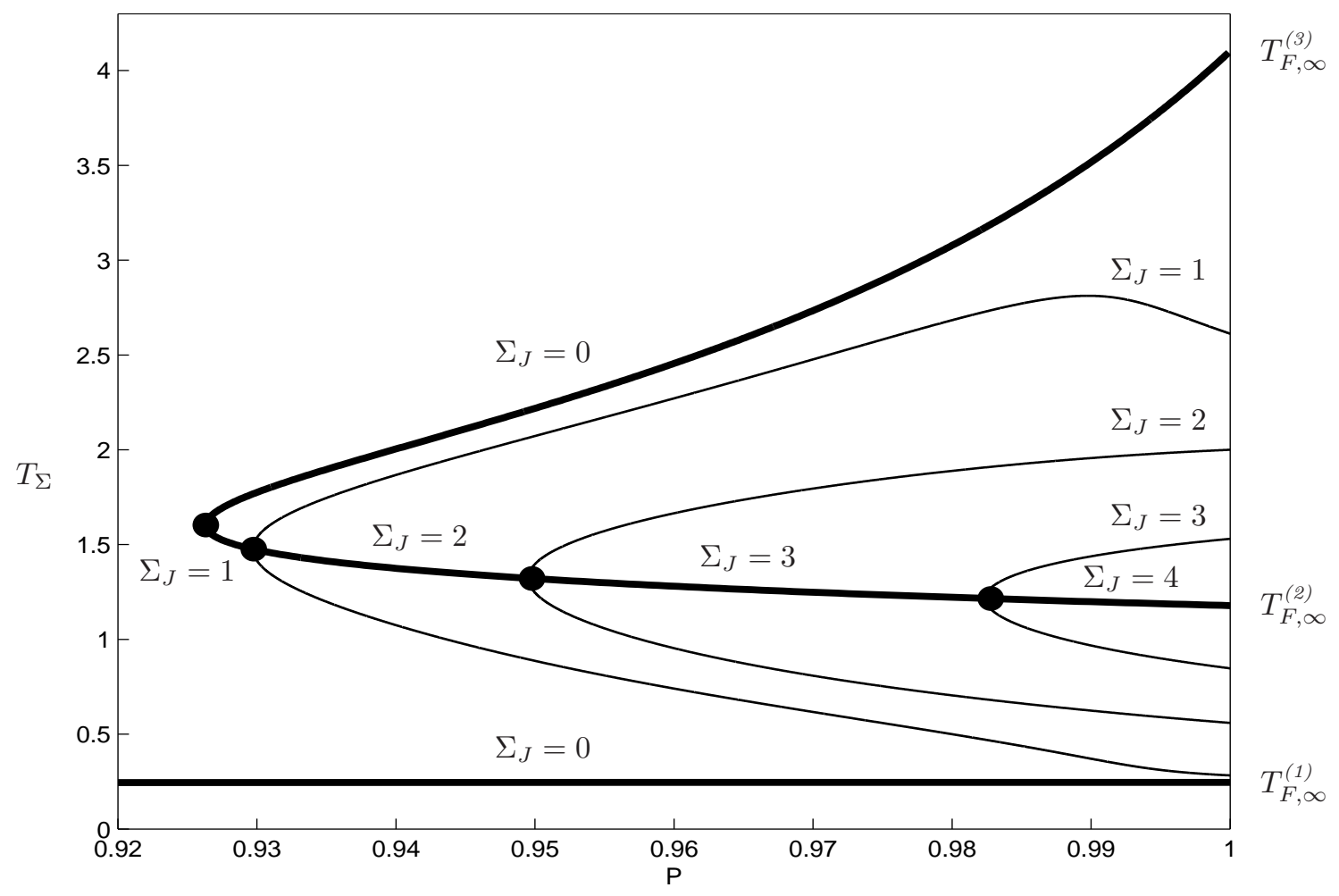

Figure 3: Bifurcation diagram for the nonlinearity parameter P. Heavy curves correspond to homogeneous solutions; normal curves correspond to heterogeneous solutions. Filled circles represent bifurcations. The left-most bifurcation is the tangent bifurcation that leads to multiple homogeneous solutions; the bifurcations from which the heterogeneous branches emerge are pitchfork bifurcations. Included also is the corresponding index $\Sigma_{J}$ (Section 4.1.3).

on the linear stability properties is closely related to the "exchange of stability principle", cf. Kielhöfer (2004).

The linear stability analysis shows that the pool-boiling problem is linearly bi-stable. The homogeneous nucleate-boiling $\left(T_{F}^{(1)}\right)$ and film-boiling $\left(T_{F}^{(3)}\right)$ states are the only stable states; other steady states are always unstable. The bi-stability implies that the system has two basins of attraction, associated with the two stable states $T_{F}^{(1)}$ and $T_{F}^{(3)}$, divided by a separatrix formed by the stable manifolds of the unstable solutions (Mischaikow (1995)).

\subsubsection{Quantitative linear stability properties}

The unstable heterogeneous solutions develop into essentially unsteady states in case of some non-zero initial perturbation $v_{0}(\boldsymbol{x})$. In practice, such perturbations are always present. Hence, the heterogeneous steady-state solutions cannot be sustained by the system and must undergo some evolution in time. This evolution depends largely on the unstable eigenmode(s) $\psi_{k}(\boldsymbol{x})$, i.e. for which the corresponding eigenvalue $\mu_{k}$ is negative, according to (18).

Figure 5 gives the sequence of eigenvalues $\mu_{1}<\mu_{2}<\ldots$ corresponding with the mode- $n$ solutions $(n=1,2,3)$. The number of negative eigenvalues equals $n=\Sigma_{J}$, consistent with the values of the index $\Sigma_{J}$ in Figure 3. Furthermore, the magnitude $\left|\mu_{k}\right|$ of the negative eigen- 


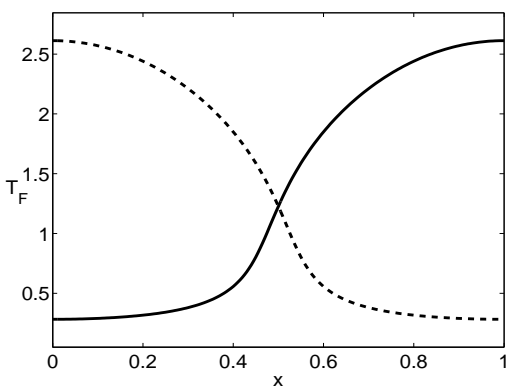

a) Pair of mode-1 solutions

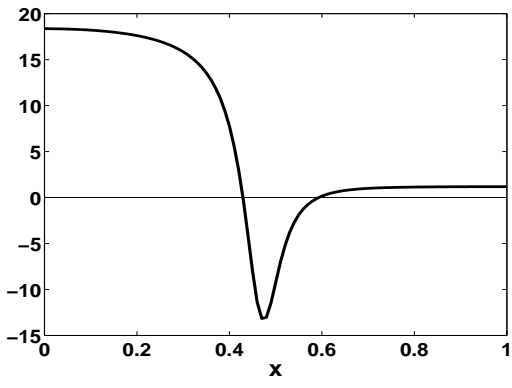

d) $\gamma\left(T_{F, \infty}\right)$ (mode-1 solution)

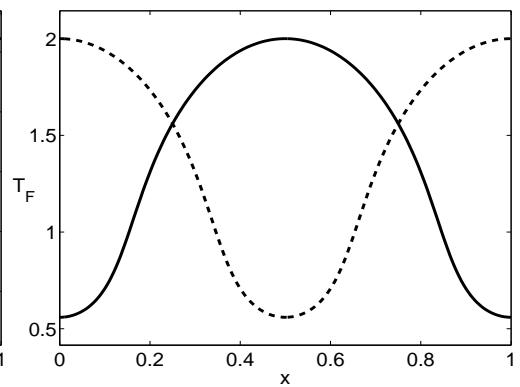

b) Pair of mode-2 solutions

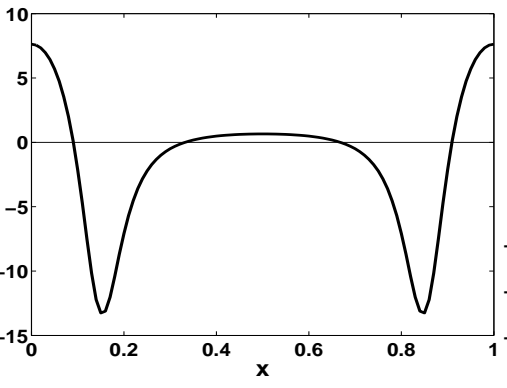

e) $\gamma\left(T_{F, \infty}\right)$ (mode-2 solution)

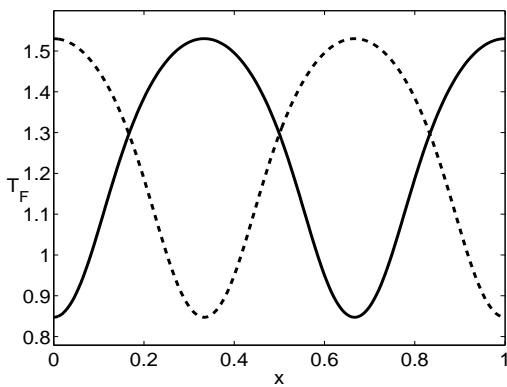

c) Pair of mode-3 solutions

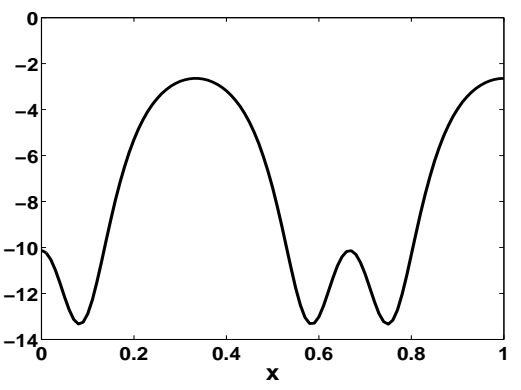

f) $\gamma\left(T_{F, \infty}\right)$ (mode-3 solution)

Figure 4: Heterogeneous steady-state (mode-n) solutions of the boiling problem. The top row gives the boundary profiles $T_{F, \infty}$ (solid) and $T_{F, \infty}^{*}$ (dashed) of the pairs of mode-n solutions. The bottom row gives coefficient $\gamma$ corresponding with $T_{F, \infty} ; \gamma<0$ indicates regions of transition boiling.

values grows - implying higher growth rates of perturbations - with increasing $n$. Figure 6 shows the boundary profiles of the unstable eigenmodes $\psi_{k}(\boldsymbol{x})$, with corresponding $\mu_{k}$-values as indicated, associated with the mode- $n$ solutions. Figure 7 gives the three unstable modes of the mode- 3 solution in the whole domain $\mathcal{D}$. Note that due to the maximum principle the extrema of $\psi_{k}$ occur at the boundary of $\mathcal{D}$. The mode- 1 solution has only one unstable eigenmode. This mode dominates the evolution of the instability, largely independent of the initial perturbation. The mode- 2 and mode- 3 solutions have multiple unstable eigenmodes with associated eigenvalues of comparable magnitude and, consequently, the space spanned by these modes has dimension larger than one. Thus the evolution of the instability becomes essentially dependent upon the initial perturbation and allows a much richer spatial structure compared to the mode-1 case.

\subsubsection{The role of heater properties in the stability behaviour}

The inherent instability of transition boiling greatly hampers reliable and reproducible laboratory experiments on boiling heat transfer under transition conditions. The heater properties are important design parameters for such transition experiments (Blum et al. (1996)). Our pool-boiling model enables examination of the role of the heater properties in the stability behaviour of pool-boiling systems under representative conditions (i.e. heterogeneous boiling states on realistic heaters) and may thus be beneficial for the design of laboratory experiments. To this end a short exploratory study, as a prelude to future work, is given below.

In the present model the heater properties are described by the parameters $\Lambda$ (thermal 


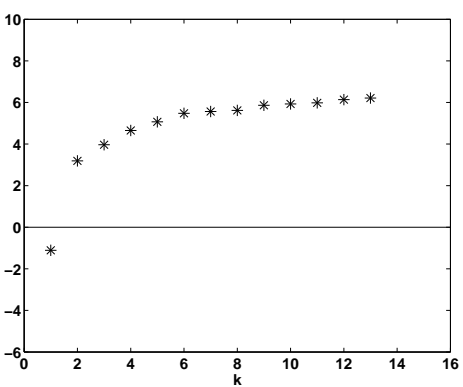

a) Mode-1 solutions

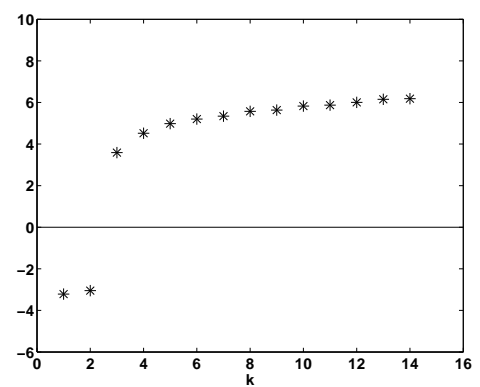

b) Mode-2 solutions

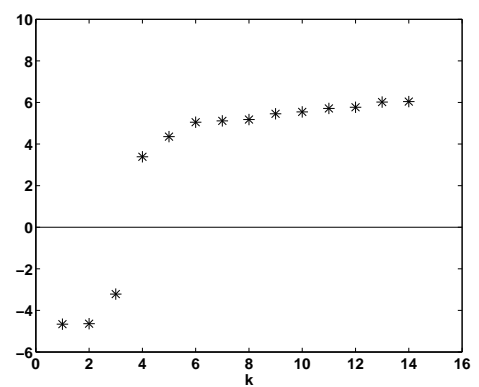

c) Mode-3 solutions

Figure 5: Sequence of eigenvalues $\mu_{1}<\mu_{2}<\ldots$ corresponding with each of the mode-n solutions. The eigenvalues are represented as $\beta_{k}=\operatorname{sign}\left(\mu_{k}\right) \ln \left|\mu_{k}\right|$ so as to enhance legibility.

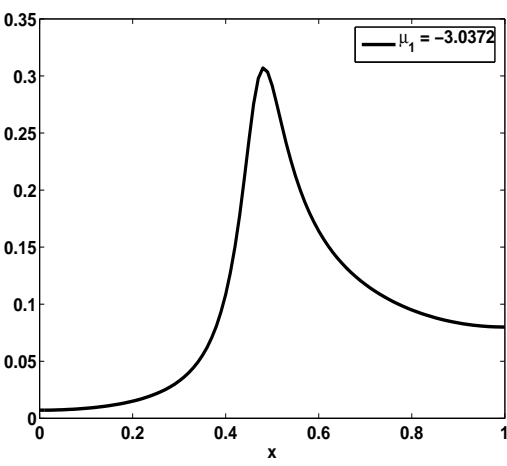

a) Mode-1 solutions

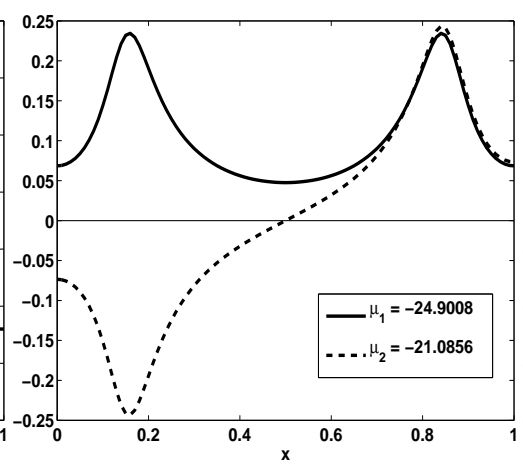

b) Mode-2 solutions

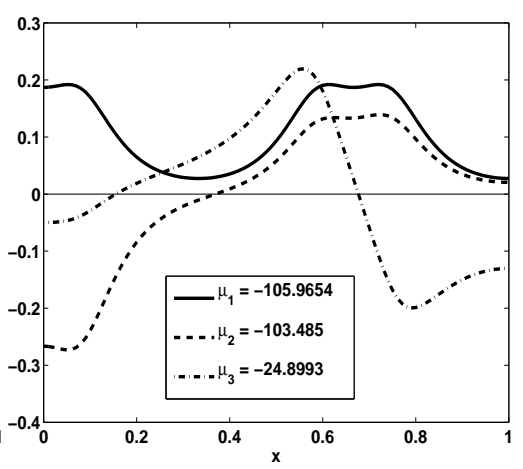

c) Mode-3 solutions

Figure 6: Unstable modes (eigenfunctions $\psi_{k}(\boldsymbol{x})$ with $\mu_{k}<0$ ) restricted to the boundary $\Gamma_{F}$, corresponding with each of the mode-n solutions.

conductivity) and $D$ (heater thickness). In the case study we investigate the changes in stability properties of the heterogeneous solutions induced by variation of $\Lambda$ or $D$. The instability is quantified in terms of the eigenvalue $\mu_{1}<0$ of the most unstable eigenmode. Figure 8 gives $\mu_{1}$ as a function of $\Lambda$ (panel $a$ ) and $D$ (panel $b$ ) for the mode- $n$ heterogeneous steady-state solutions (symbols), the homogeneous transition solution $T_{F}^{(2)}$ (with $\mu_{1}=z_{1}^{-}$as in Theorem 2) and the lower bound $\mu_{*}$ according to (28). The dashed vertical line indicates the parameter value used in the case study. (The plots actually show $-\mu$ so as to facilitate the logarithmic scale.) Both graphs reveal that the number of mode- $n$ solutions decreases both with increasing $\Lambda$ and increasing $D$. Beyond $\Lambda \approx 1.2$ mode- $n$ solutions have vanished altogether; beyond $D \approx 0.1$ the system settles for three mode- $n$ solutions and effectively becomes independent of the heater thickness for $D \gtrsim 0.5$. Thus increasing thermal conductivity and/or the heater thickness promotes homogeneity and uniqueness of boiling states. This is consistent with results in Speetjens et al. (2006a).

In Figure $8 a$ one can observe the bifurcation of mode- $n$ profiles from the $T_{F}^{(2)}$-profile with decreasing thermal conductivity $\Lambda$. (For $D$ essentially the same happens. This is less apparent in Figure 8b, though.) The instability of the mode- $n$ solutions is stronger (in the sense that $\left|\mu_{1}\right|$ increases) for larger $n$ and $T_{F}^{(2)}$ appears to be the most unstable solution. Physically, this may be explained by the fact that the portion of the interface on which the temperature is in 

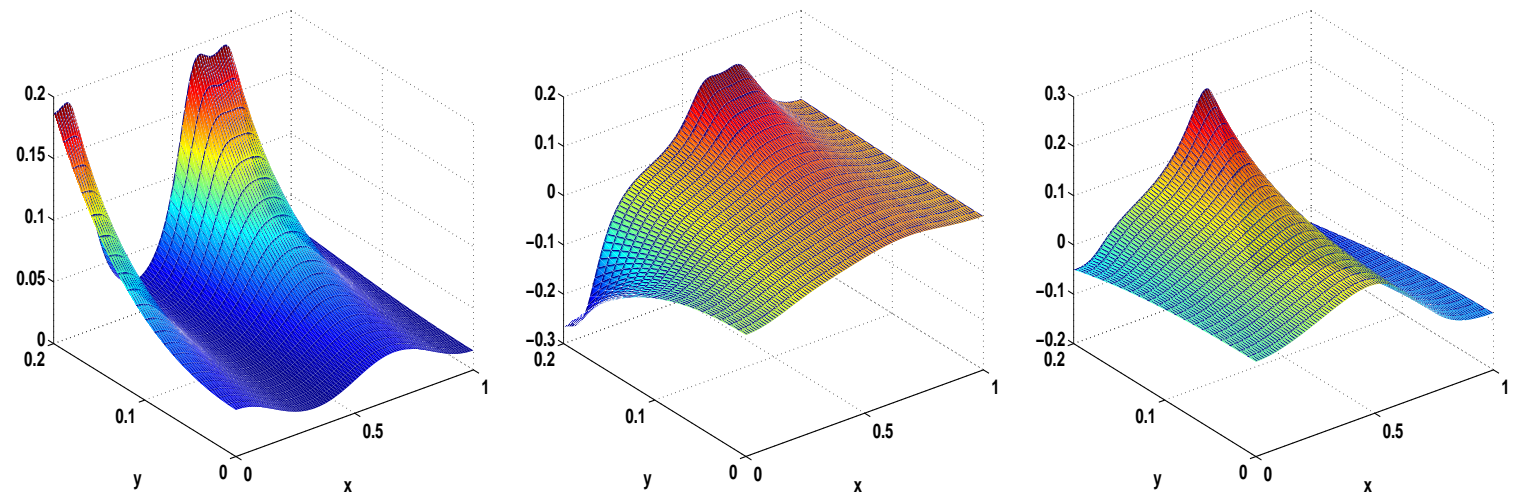

a) $\psi_{1}(\boldsymbol{x}), \mu_{1}=-106.0$

b) $\psi_{2}(\boldsymbol{x}), \mu_{2}=-103.5$

c) $\psi_{3}(\boldsymbol{x}), \mu_{3}=-24.9$

Figure 7: The three unstable modes of the mode-3 solution in the domain $\mathcal{D}$.

the transition regime (i.e. where $\gamma<0$ ) grows with $n$ and is maximal for the homogeneous state $T_{F}^{(2)}$.

The mode- $n$ solutions for $n>1$ become more unstable (larger $\left|\mu_{1}\right|$ ) for increasing $\Lambda$ and/or $D$. For the homogeneous solution $T_{F}^{(2)}$, on the other hand, this lessens the instability. For the mode- 1 solution $\left|\mu_{1}\right|$ exhibits a non-monotonic dependence on $\Lambda$ and on $D$. These observations reveal that the dependence of the stability behaviour on changes in the heater properties is related to the kind of steady-state solution. For the mode- $n(n>1)$ solutions, increasing heater thickness $D$ and/or thermal conductivity $\Lambda$ amplifies instability, whereas for the homogeneous solution $T_{F}^{(2)}$ this dampens instability.

The above results strongly suggest that, despite significant quantitative variations in $\mu_{1}$, the instability itself remains under all conditions and that, in consequence, the existence of stable heterogeneous solutions for specific heater properties is therefore highly unlikely. Laboratory experiments support this assertion (consult Blum et al. (1996) for a survey). This consolidates the widely-accepted observation that active stabilisation, via e.g. the methodology by Auracher \& Marquardt (2004), is essential for detailed experimental studies on homogeneous boiling states in the transition region and on any heterogeneous boiling state. Moreover, this confirms that the stability analysis of Haramura (1991) is erroneous, as already pointed out by Blum \& Marquardt (1998). Haramura (1991) studied the stability of homogenous boiling states on the fluid-heater interface of a three-dimensional heater with constant heating in the transition boiling regime (i.e. $\gamma<0$ ) and derived critical conditions for which the homogeneous boiling state supposedly becomes unstable. These critical conditions are in contradiction with findings in previous studies (e.g. Blum et al. (1996), Stephan (1965)) as well as with those in the present study.

WM: Verweis auf Arbeit von Haramuru aufnehmen?

\subsection{Evolution of perturbed unstable steady-state solutions}

The nonlinear long-term evolution of perturbed unstable steady-state solutions, i.e. the nonlinear (in)stability behaviour, can be determined via numerical simulation of (1). We used a spectral tau method based on Fourier $(x)$ and Chebyshev $(y)$ expansion of $T(\boldsymbol{x}, t)$ for spatial discretisation in combination with a second-order Crank-Nicholson time-marching 

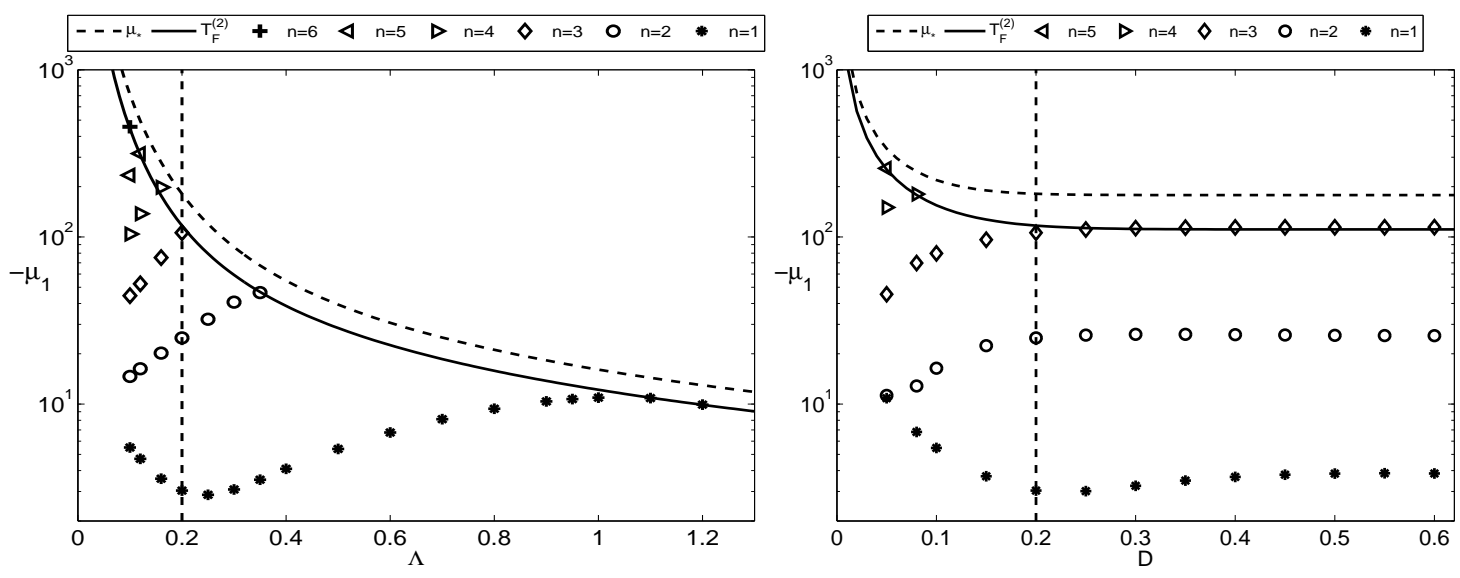

a) Thermal conductivity $\Lambda$

b) Thickness D

Figure 8: Effect of varying heater properties ( $\Lambda$ and $D)$ upon the stability properties. Shown is the eigenvalue $\mu_{1}<0$ of the most unstable eigenmode for the mode-n solutions (symbols), the homogeneous transition solution $T_{F}^{(2)}$ and the lower bound $\mu_{*}$ according to (28). The dashed vertical line indicates the parameter value used in the case study.

scheme (Canuto et al. (1987)). The nonlinearity on the interface $\Gamma_{F}$ has been dealt with by Picard iteration (Kreyszig (1999)).

Numerical studies of (1) serve two purposes, namely validation of the linear stability analysis (Section 3.2) and gaining first insight into the nonlinear stability behaviour of the pool-boiling system. These two topics are addressed in Section 4.2.1 and Section 4.2.2, respectively.

\subsubsection{Validation of the linear stability analysis}

The spectral scheme proposed above is used for the numerical simulation of the linearised heat-transfer model (9). Tests with various identical initial conditions $v_{0}(\boldsymbol{x})$ for each of the steady-state solutions $T_{\infty}$ reveal that solutions $v(\boldsymbol{x}, t)$ obtained through the linear model (9) and the expansion (18) coincide within machine accuracy. This validates the eigenmode decomposition (18).

A second issue is a comparison of the evolution of the perturbation $v(\boldsymbol{x}, t)$ in the nonlinear model (1) and in the eigenmode decomposition (18). Tests reveal that in both cases the stability properties are qualitatively the same: heterogeneous steady-state solutions are unstable and the homogeneous nucleate-boiling and film-boiling states are stable. This provides strong evidence that the pool-boiling system is nonlinearly bi-stable as well.

Quantitative validation of the linear stability analysis and establishment of a range of validity of the linear approximation follows from investigation of two representative heterogeneous cases, namely the steady-state mode- 1 and mode- 2 solutions. As initial perturbation we take $v_{0}(\boldsymbol{x})=\epsilon \psi_{1}(\boldsymbol{x})$, with $\epsilon=0.01$ and $\psi_{1}$ the first eigenfunction (normalised) of (13)-(15). Figure 9 shows the profiles at the interface $\Gamma_{F}$ at several time instances $t$ corresponding with the solutions to the nonlinear model and the linearised problem. (For the nonlinear model we give the departure $v(\boldsymbol{x}, t)=T(\boldsymbol{x}, t)-T_{\infty}(\boldsymbol{x})$ from the initial state.) The more pronounced peaks correspond with more advanced time levels. The results show a good agreement be- 
tween linear approximation and nonlinear evolutions for a significant time interval.

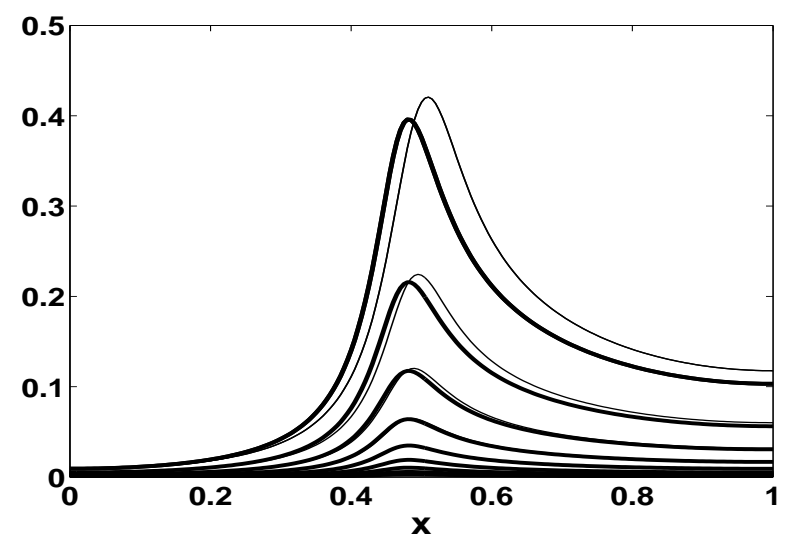

a) Mode-1 solution

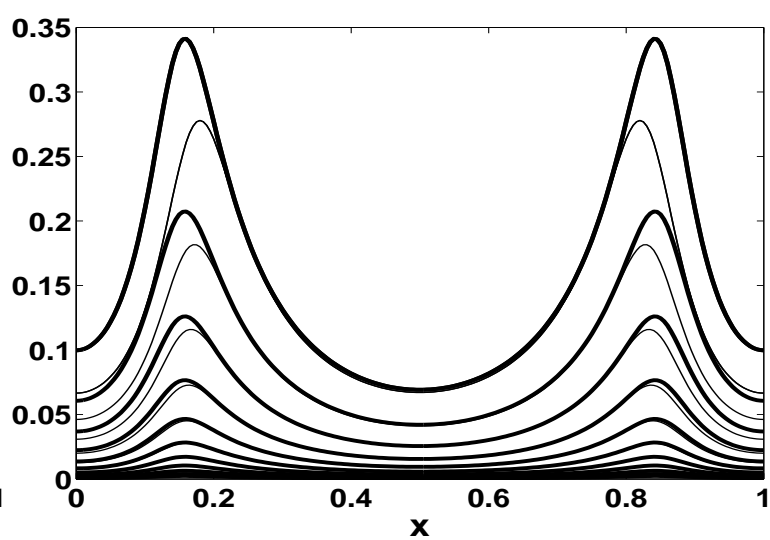

b) Mode-2 solution

Figure 9: Solutions $v(\boldsymbol{x}, t)$ of the linear approximation((12)) (heavy) and of the nonlinear model (solid) for the mode-1 (panel a) and mode-2 (panel b) steady-state solutions. Initial perturbation is $v_{0}(\boldsymbol{x})=0.01 \psi_{1}(\boldsymbol{x})$. The curves correspond to time steps $\Delta t=5$ (mode-1) and $\Delta t=0.5$ (mode-2).

\subsubsection{Nonlinear stability analysis}

The present case study involves the following unstable steady-state solutions: the homogeneous solution $T_{F, \infty}^{(2)}$ in the transition-boiling regime and the three pairs of mode- $n$ solutions. Perturbations are not imposed explicitly but due to rounding errors in the machine representation of the initial condition. This implies a machine-dependent yet reproducible perturbation. These small perturbations are sufficient to trigger evolution of the unstable states. We use the functionals $T_{\Sigma}=\sum_{n} \widetilde{T}_{n}$ and $\mathcal{K}(k \Delta t)=\left\|\mathbb{T}^{k}-\mathbb{T}^{k-1}\right\| /\left\|\mathbb{T}^{k}\right\|$, where $\mathbb{T}^{k}$ is the matrix consisting of all coefficients in the discrete Fourier-Chebyshev series of the solution at $t=k \Delta$, as measures for quantifying the evolution. The mode- $n$ solutions always occur as pairs (41). We only consider one solution of this pair (lower legs of the heterogeneous branches in Figure 3).

Figure $10 a$ demonstrates the dynamical behaviour of the system during transition from unstable to stable steady states via the measure of unsteadiness $\mathcal{K}(t)$ for the unstable homogeneous solution $T_{F, \infty}^{(2)}$. The progression clearly reveals that the evolution of the temperature field accelerates (i.e. $\mathcal{K}(t)$ grows continuously) up to a turning point at $t \approx 6$, where the situation reverses and a deceleration sets in that continues until a stable steady state is reached at $t \approx 25$. (The erratic evolution beyond $t \approx 25$ is due to fluctuations around the steady state at machine-accuracy level.) The mode- $n$ solutions exhibit essentially similar behaviour as that shown in Figure 10a; differences are entirely quantitative in that turning points and attainment of stable steady-states occur at different instances in time. Figure $10 b$ gives the evolution from unstable to stable steady-states in terms of the functional $T_{\Sigma}(t)$ (split up into two frames.) The unstable homogeneous solution $T_{F, \infty}^{(2)}$ and the mode-3 solutions progress towards the stable steady-state solution $T_{F, \infty}^{(1)}$ in the nucleate-boiling regime (lower dashed line); the mode- 1 and mode- 2 solutions progress towards the stable steady-state solution $T_{F, \infty}^{(3)}$ in the film-boiling regime (upper dashed line). The sharp transition of the evolutions occur around the beforementioned turning points and reflect the fact that the changeover from 
unstable to stable states happens rather abruptly within a relatively narrow time window. Moreover, this changeover takes place earlier, suggesting stronger instability, with decreasing length scales of the heterogeneous features (higher $n$ ) of the mode- $n$ solutions. This is in qualitative agreement with experimental observations (Dhir (1998)).

Figure 11 gives the evolutions of the profiles of the interface temperature $T_{F}$ for each of the unstable steady-state solutions in Figure $10 b$ at equidistant time intervals, where the arrows indicate progression in time. (Time intervals are different for each case and set proportional to the duration of the entire evolution.) The heavy dashed profiles correspond with the intermediate state at the respective turning points; the lower and upper dashed lines indicate the stable nucleate-boiling and film-boiling states, respectively. The evolution of the profiles nicely illustrate the progression towards either the nucleate-boiling state (panels $a$ and $d$ ) or the film-boiling state (panels $b$ and $c$ ). Note that for the unstable homogeneous case $T_{F, \infty}^{(2)}$ (panel $a$ ) the profile remains homogeneous throughout the evolution. Moreover, the expansion and subsequent condensation of the profiles before and after the turning point (heavy dashed) demonstrates the initial acceleration and the subsequent deceleration of the evolution. The acceleration phase of the mode- $n$ solutions is characterised by rapid smoothing of the heterogeneous features (panels $b-d$ ); during the subsequent deceleration phase the (approximately) homogeneous intermediate state gradually tends to the ultimate stable state.

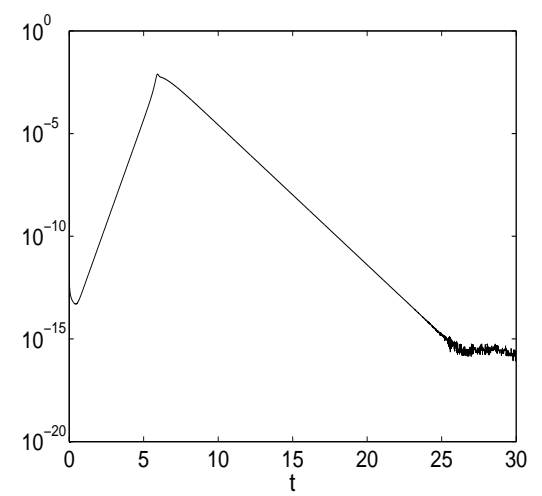

a) $\mathcal{K}(t)$ for $T_{F, \infty}^{(2)}$.
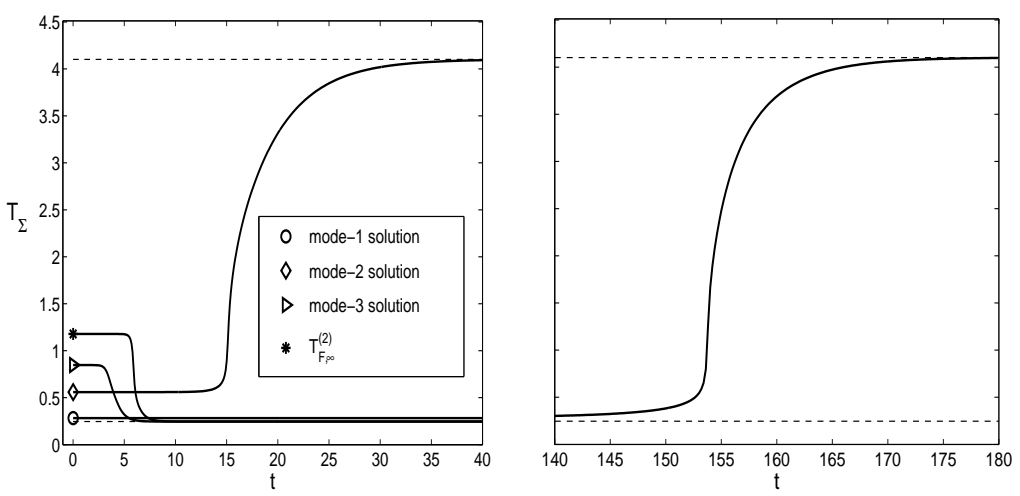

b) Progression towards one of the two stable steady states.

Figure 10: Evolution of perturbed unstable steady-state solutions towards a stable state. Panel a shows the initial acceleration and subsequent deceleration of the evolution with the measure of unsteadiness $\mathcal{K}(t)$ for the unstable homogeneous solution $T_{F, \infty}^{(2)}$. Panel b gives the progression of the unstable states (as indicated) towards one of the two stable steady states (dashed) in terms of the functional $T_{\Sigma}(t)$. (Split-up into two frames; the right frame concerns only the mode-1 solution.) Only the parent solution $T_{F}$ of each conjugate pair (41) is included.

\section{Conclusions}

In this paper we consider a 2D nonlinear heat-transfer problem as a model for pool-boiling systems. The model problem involves only the temperature distribution within the heater and models the heat exchange with the boiling medium via a nonlinear boundary condition imposed at the fluid-heater interface. This results in a linear parabolic partial differential equation (heat equation) with a nonlinear Neumann boundary condition at the fluid-heater 
interface. Important information about the (qualitative) behaviour of this dynamical system can be obtained from its steady-state solutions and the corresponding stability properties. The steady-state behaviour has been studied in Speetjens et al. (2006a). The main topic of the present study is the corresponding stability behaviour. To this end a linear (short-term) and nonlinear (long-term) stability analysis are performed.

In the linear stability analysis the linearised heat-transfer model is, by separation of space and time, reduced to a nonlinear eigenvalue problem that depends only on the two spatial variables. Separation of the two spatial variables subsequently leads to a nonlinear spatially one-dimensional problem for the eigenvalues. Analysis of the latter problem yields generic stability properties for steady-state solutions. These are demonstrated and validated by numerical simulations in a representative case study. One of the main conclusions is that the (linearised as well as nonlinear) system is bi-stable: all steady-state solutions, except the homogeneous nucleate-boiling and film-boiling states, are inherently unstable. Perturbed unstable states always progress towards one of these two stable states. Our study furthermore strongly suggests that these stability properties are qualitatively independent of heater properties (thermal conductivity $\Lambda$ and thickness $D$ ). Changes in heater properties affect the stability properties of the system only in a quantitative manner. Thus the present study rigorously demonstrates the bi-stability of pool-boiling systems, which is consistent with laboratory experiments (Auracher \& Marquardt (2004)) and other theoretical studies (Blum et al. (1996), Blum et al. (1999), Gurevich \& Mints (1987), Kovalev \& Usitakov (2003).

The numerical simulations for the case study provide evidence that there is a strong analogy between the nonlinear heat-transfer problem and generic nonlinear parabolic evolution equations, which typically have a nonlinearity in the partial differential equation. This analogy suggest the fundamental property that the dynamical behaviour of the system is dominated by a global attractor made up of steady-state solutions and corresponding heteroclinic connections (Section 3.1). Both the linear and nonlinear stability behaviour that we observe in the pool-boiling system indicate that this property holds. Although many stability results, based on both theoretical analysis and numerical experiments, are derived in this paper, a complete rigorous mathematical analysis of the dynamics of the pool-boiling model is not yet available.

Recent studies revealed that the steady-state behaviour and the mathematical structure of three-dimensional pool-boiling problems is essentially similar to that of the simplified 2D case considered here (Speetjens et al. (2006b)). This means that the stability behaviour found in the present work in principle extends to the three-dimensional case. Moreover, the present analysis may form the basis for future research on active stabilisation of unstable heterogeneous boiling states by extending the model with a temperature-control loop similar to that proposed in Blum et al. (1996).

\section{References}

Adiutori, E.F. 1964 New theory of thermal stability in boiling systems. Nucleonics, 22, 92-101.

Auracher, H. \& Marquardt, W. 2004 Heat transfer characteristics and mechanisms along entire boiling curves under steady-state and transient conditions. J. Heat Fluid Flow, 25, 223-242. 
Blum, J., Marquardt, W. \& Auracher, H. 1996 Stability of boiling systems. Int. J. Heat Mass Transfer, 39, 3021-3033.

Blum, J. \& Marquardt, W. 1998 Objection to Haramura's criterions for temperature uniformity across the surface in transition boiling. Internal Report, LPT, RWTH Aachen.

Blum, J., LÜttich, T. \& Marquardt, W. 1999 Temperature wave propagation as a route from nucleate to film boiling? In Proceedings of the Second International Symposium on Two-Phase Flow Modelling and Experimentation, Rome, Vol 1 (ed. G.P. Celata, P. DiMarco \& R.K. Shah), Edizioni ETS, Pisa.

Canuto, C., Hussaini, M. Y., Quarteroni, A. \& Zang, T. A. 1987 Spectral Methods in Fluid Dynamics. Springer, Berlin.

DhIR, V. K. 1998 Boiling heat transfer. Ann. Rev. Fluid Mech. 30, 365-401.

Fife, P. C. 1979 Mathematical Aspects of Reacting and Diffusing Systems, Springer, Berlin.

Govaerts, W. J. F. 2000 Numerical methods for Bifurcations of Dynamical Equilibria, SIAM, Philadelphia.

Gurevich, A. V. \& Mints, R. G. 1987 Self-heating in normal metals and superconductors. Rev. Mod. Phys. 59, 941-999.

Haramura, Y. 1991 Temperature uniformity across the surface in transition boiling. ASME J. Heat Transfer, 113, 980-984.

Iooss, G. \& Joseph, D. D. 1990 Elementary Stability and Bifurcation Theory. Springer, Berlin.

KIElhöfer, H. 2004 Bifurcation Theory. An Introduction with Applications to PDEs. Springer, New York.

Kovalev, S.A. 1968 On methods of studying heat transfer in transition boiling. Int. J. Heat Mass Transfer, 11, 279-283.

Kovalev, S. A. \& Rybchinskaya, G. B. 1978 Prediction of the stability of pool boiling heat transfer to finite disturbances. Int. J. Heat Mass Transfer, 21, 691-700.

Kovalev, S. A. \& Usitakov, S. V. 2003 Analysis of the stability of boiling modes involving the use of stability diagrams. High Temp., 41, 68-78.

Kreyszig, E. 1999 Advanced Engineering Mathematics, Wiley, Chichester.

Maier-PaApe, S. \& Miller, U. 2002 Path-following the equilibria of the Cahn-Hilliard equation on the square. Comput. Visual Sci., 5, 115-1138.

Maier-PaApe, S., Mischaikow, K. \& Wanner, T. 2005 Structure of the attractor of the Cahn-Hilliard equation on a square. To appear in International J. Bifurcation Chaos

Mischaikow, K. 1995 Global Asymptotic dynamics of gradient-like bistable equations. SIAM J. Math. Anal., 26, 1199-1224. 
MudawAR, I. 2001 Assessment of high-heat-flux thermal management schemes. IEEE Transactions-CPMT: Components and Packaging Technologies, 24, pp. 122-141.

VAN Ouwekerk, H. 1972 Burnout in pool boiling. The stability of boiling mechanisms. Int. J. Heat Mass Transfer, 15, pp. 25-33.

PAo, C. V. 1992 Nonlinear Parabolic and Elliptic Equations. Plenum, New York.

Speetuens, M., Reusken, A. \& Marquardt, W. 2006a Steady-state solutions in a nonlinear pool-boiling model. Commun. Nonlinear Sci. Numer. Simul., doi:10.1016/j.cnsns.2006.11.001.

Speetjens, M., Reusken, A. \& Marquardt, W. 2006b Steady-state solutions in a threedimensional nonlinear pool-boiling heat-transfer model. Commun. Nonlinear Sci. Numer. Simul., doi:10.1016/j.cnsns.2006.11.002.

Stephan, K. 1965 Stabilität beim Sieden. Brennst.-Wäme-Kraft, 17, 571-578.

Temam, R. 1997 Infinite-dimensional Dynamical Systems in Mechanics and Physics. Springer, New York.

Thome, J.R. 2003 Boiling. In Handbook of Heat Transfer (ed. A. Bejan \& A. D. Krause), Wiley \& Sons, Hoboken, pp. 635-717.

\section{Appendix: heat-flux function}

The heat-flux function $q_{F}\left(Z ; \Pi_{2}, \Pi_{3}\right)$ is given by

$$
\begin{aligned}
q_{F}(Z) & =h(Z) Z, \quad \text { with } \\
h(Z) & =C_{D}\left\{F_{1}-F_{2} H\left(C_{D} Z-1\right)\right\}, \quad H(\zeta)=\frac{1}{2}\left[\tanh \left(\frac{2 \zeta}{W}\right)+1\right] .
\end{aligned}
$$

The function $H(\zeta)$ is a smoothed Heaviside function. The parameter $W>0$ controls the width of the transient (from $H=0$ to $H=1$ ) around $\zeta=0$ and is specified a priori. The value of $W$ indirectly sets the physical parameter $\Pi_{3}$. The coefficient $C_{D}$ rescales the argument $Z$ such that the single deflection point of $q_{F}$ coincides with $Z=1$, i.e., $q_{F}^{\prime \prime}(1)=0$. Its value is defined implicitly through

$$
2 \frac{d H}{d \zeta}\left(C_{D}-1\right)+C_{D} \frac{d^{2} H}{d \zeta^{2}}\left(C_{D}-1\right)=0,
$$

and thus depends only on $W$. It can be shown that $q_{F}$ as in (48) possesses a local maximum and minimum at $Z_{\max }<1$ and $Z_{\min }>1$, respectively. Introduction of the scaling factors $F_{1}$ and $F_{2}$, which scale $q_{F}$ such that the conditions

$$
q_{F}^{\prime}\left(Z_{\max }\right)=0, \quad q_{F}^{\prime}\left(Z_{\min }\right)=0, \quad q_{F}\left(Z_{\max }\right)=1, \quad q_{F}\left(Z_{\min }\right)=\Pi_{2}^{-1},
$$

are fulfilled, then results in a heat-flux function that is consistent with the physical boiling curve. For given $\Pi_{2}$ and $W$ the conditions (49) result in four nonlinear equations that can be solved for the four unknowns $\left(F_{1}, F_{2}, Z_{\min }, Z_{\max }\right)$. 

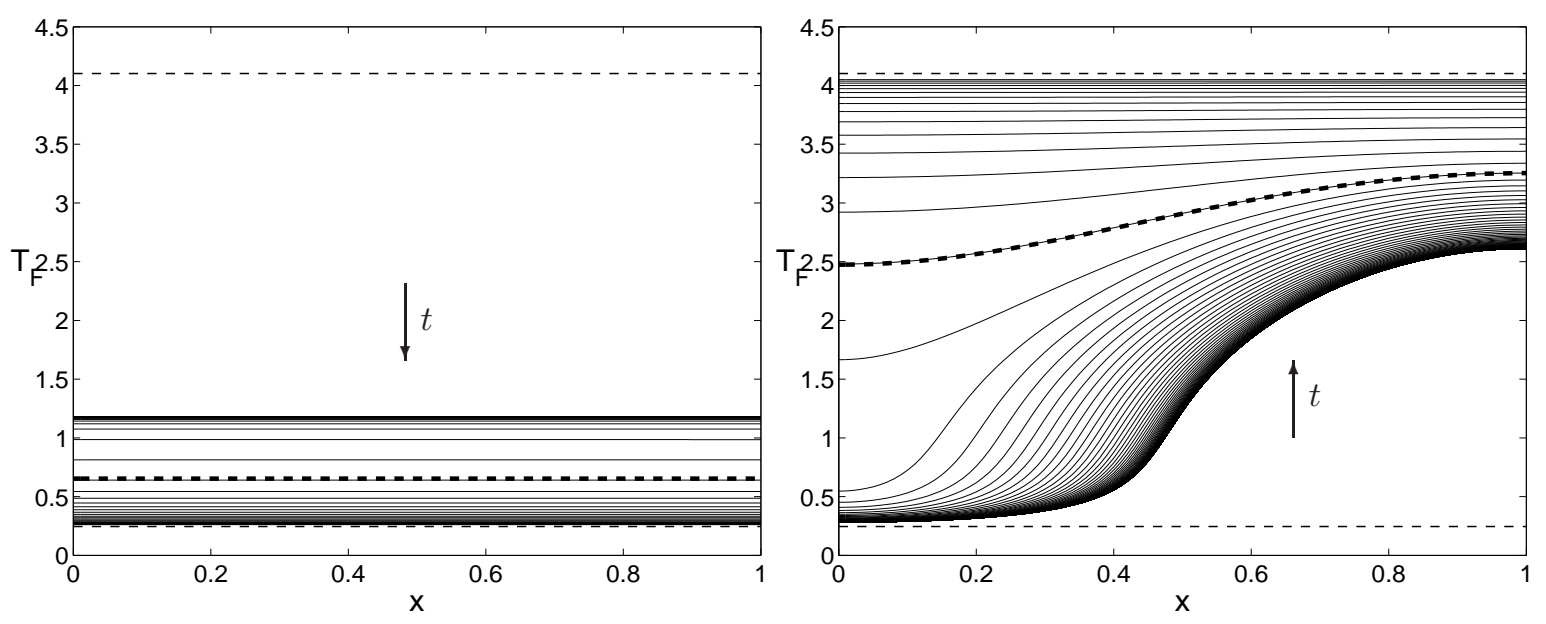

a) unstable homoqeneous solution.

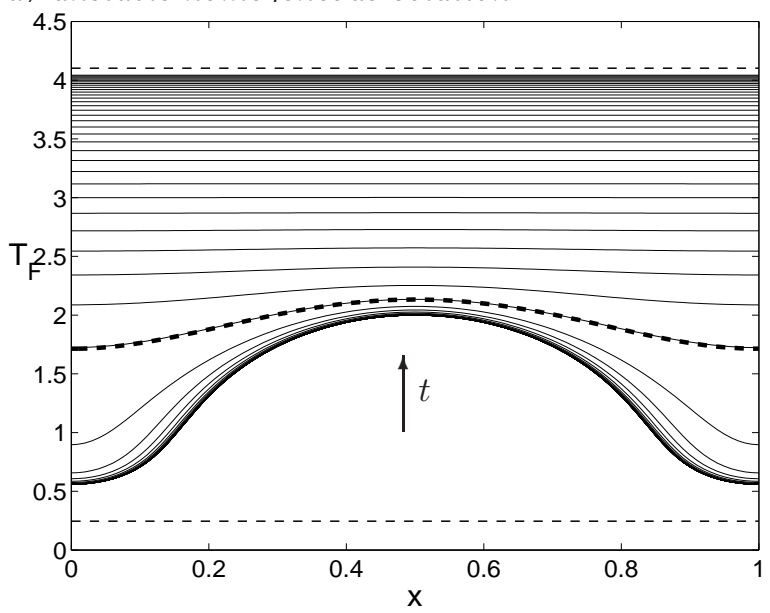

b) Mode-1 solution.

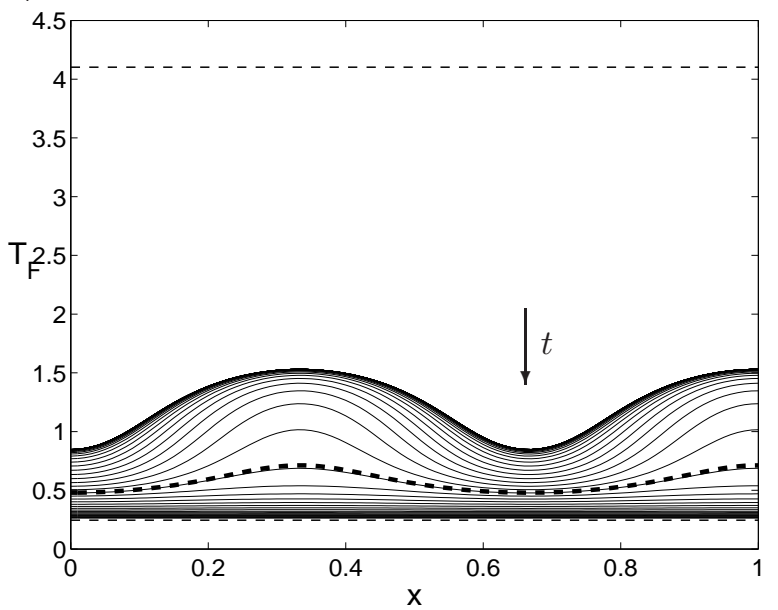

c) Mode-2 solution.

d) Mode-3 solution.

Figure 11: Evolution of the perturbed unstable steady-state solutions (heavy) on the interface. Shown are the progressions of the boundary profiles (equidistant time intervals) towards one of the two stable steady states. The arrow indicates progression in time. The heavy dashed profiles indicate the intermediate state at the turning points; the lower and upper dashed lines indicate the stable nucleate-boiling and film-boiling states, respectively. Only one solution $T_{F}$ of each conjugate pair (41) is included. 